\title{
Une analyse informationnelle de la crise financière récente
}

\author{
Jean-Pierre Allegret* \\ Camille Cornand $^{* *}$
}

13 décembre 2013

\section{Résumé :}

Nous proposons une analyse informationnelle de la crise récente en s'appuyant sur la littérature et en adoptant une double perspective. D'une part, nous analysons les difficultés liées à la capacité des agents économiques et financiers à traiter l'information et au manque d'incitations de ces mêmes acteurs de marché à rechercher l'information dans le système financier. L'hypothèse de myopie au désastre est mobilisée pour expliquer pourquoi les participants au marché ne tiennent pas compte des informations pertinentes. Cette hypothèse permet d'expliquer notamment le boom sur le marché des subprimes. Une telle bulle a également pu être interprétée en termes d'insuffisante production d'information par les agents privés. Enfin, l'éclatement de la bulle peut être compris comme la réaction des participants au marché à une situation de connaissance commune d'un mauvais état fondamental. D'autre part, nous analysons la réponse des autorités en tant que pourvoyeur d'information face aux difficultés du marché à internaliser les externalités négatives dues aux déficiences en matière de production et de traitement de l'information. Nous analysons les réponses à la crise formulées par le régulateur en matière de communication : face à l'instabilité bancaire, une solution envisagée a été de proposer des tests de stress; en matière de gestion des anticipations, les banques centrales ont été amenées à utiliser des politiques monétaires non-conventionnelles. Dans les deux cas, la politique informationnelle du régulateur joue un rôle central et interagit stratégiquement avec la réaction du marché.

Title : An informational analysis of the recent financial crisis

\begin{abstract}
:
Relying on the recent literature, we propose an informational interpretation to the recent financial crisis. We adopt a twofold point of view. On the one hand, we analyze the difficulties associated with the inability of economic and financial agents to deal with information and with the lack of incentives for those market participants to produce information in the financial system. The disaster myopia hypothesis allows us to explain why market participants do not account for the relevant information. This assumption can illustrate the boom on the subprime market. Such a boom can also be interpreted in terms of an insufficient production in private information. Finally, the bust can be understood as the reaction of market participant to a situation of common knowledge about a bad fundamental state. On the other hand, we analyze the response of the authorities as an information provider to the difficulties of the market to internalize the negative externalities coming the deficiency in terms of information treatment and production. We analyze the regulator's responses to the financial crisis in terms of communication: with respect to banking instability, stress tests have been considered as a solution; with respect to the management of expectations, central banks have increasingly used unconventional monetary policies. In both cases, the informational policy of the regulator plays a crucial role and strategically interacts with market reaction.
\end{abstract}

Mots-clés : information, crise financière, myopie au désastre, sur-réaction, tests de stress, politique monétaire non-conventionnelle.

Keywords : information, financial crisis, disaster myopia, over-reaction, stress tests, unconventional monetary policy.

Classification JEL : F33, G01, G14, G28. 


\section{1 - Introduction}

Une crise financière décrit une situation dans laquelle l'effondrement de certaines institutions financières et de certains marchés financiers est tel qu'il conduit à des pertes massives en termes de croissance. La crise financière récente n'est à cet égard pas une exception comme le suggère la persistance d'un chômage élevé dans les principaux pays développés. Cette crise - à l'instar des crises de change des années 1990 - a semblé largement imprévisible à la fois du point de vue des investisseurs privés et des institutions internationales. Pourtant, des indicateurs avancés ont pu être identifiés ex post, suggérant l'imminence de la crise (Borio et Drehmann [2009]) : l'explosion (boom) du crédit et des prix des actifs (actions et immobilier). Les investisseurs semblent dès lors sous-estimer, et même ignorer, le risque d'effondrement financier pendant les périodes d'expansion. Au contraire, dès lors que la crise était enclenchée, tout signal négatif sur l'état de l'économie ou de certaines institutions financières a pu servir de point focal aggravant la crise.

Dans cette perspective, cet article propose une grille d'analyse des questions informationnelles qui se sont posées lors de la crise récente. Nous cherchons plus précisément à expliquer en quoi l'information est pertinente pour comprendre la crise de 2008 (et plus généralement les crises financières) à travers son impact sur le marché et le jeu stratégique qu'elle implique dès lors que les autorités tentent de répondre à ce déficit informationnel. L'insuffisance de l'information pertinente communiquée au marché et le manque de transparence sont souvent considérés comme figurant parmi les principaux éléments déclencheurs des crises financières. Une telle insuffisance de l'information est cause de déstabilisation d'une part, parce que la capacité du marché à maintenir la discipline de marché est entravée par le manque de fiabilité de l'information disponible ; et, d'autre part, parce que l'insuffisance de l'information peut conduire à des paniques. Dans les deux cas, le résultat est sous-optimal du point de vue social.

Une double perspective est adoptée ici. D'une part, nous cherchons à établir le lien entre information et comportement des investisseurs. D'autre part, nous considérons la réponse des autorités - entendues ici comme les banques centrales et les autorités de régulation - et son interaction avec le comportement des investisseurs. Les deux points de vue sont complémentaires en ce sens qu'il existe un jeu stratégique entre, d'un côté, les participants au marché, et, d'un autre côté, les autorités. Au sein de ce jeu stratégique, les autorités cherchent à influencer le comportement des agents privés en diffusant des informations sur leur propre stratégie et sur les conditions du marché.

L'article est structuré de la façon suivante. La deuxième section est consacrée à la problématique de l'information sur les marchés. Nous analysons ici les difficultés liées à la capacité des agents économiques et financiers à traiter l'information, au manque d'incitations de ces mêmes acteurs de marché à rechercher l'information dans le système financier, et aux effets des annonces sur les marchés. Ainsi, l'hypothèse de myopie au désastre est mobilisée pour expliquer pourquoi les 
participants au marché ne tiennent pas compte des informations pertinentes. Cette hypothèse permet notamment d'expliquer le boom sur le marché des subprimes ${ }^{1}$. Une telle bulle a également pu être interprétée en termes d'insuffisante production d'information par les agents privés. Nous passons en revue la littérature sur ce thème. Enfin, l'éclatement de la bulle peut être compris comme la réaction des participants au marché à une situation de connaissance commune d'un mauvais état fondamental. Nous nous appuyons sur la littérature des jeux de concours de beauté keynésien pour mettre ce mécanisme en évidence et l'illustrons au moyen d'exemples d'annonces ou de publications. Cette section permet de montrer en quoi l'information a pu jouer un rôle dans la formation de la bulle et son éclatement.

La troisième section se concentre sur la façon dont les autorités ont réagi face aux problèmes informationnels dans la crise et l'interaction entre leur réponse et le comportement du marché. Nous analysons ainsi la réponse des autorités en tant que pourvoyeur d'information face aux difficultés du marché à internaliser les externalités négatives dues aux déficiences des marchés en matière de production et de traitement de l'information. Plus précisément, deux réponses sont distinguées. En premier lieu, nous analysons la stratégie adoptée par les autorités de régulation bancaire pour diffuser des informations sur la santé des banques. Face à l'instabilité bancaire, une solution envisagée a été en effet de proposer des tests de stress. Nous nous concentrons sur la question de la diffusion des résultats des tests de stress en matière de stabilité financière. En second lieu, les banques centrales ont été conduites à accentuer leur politique de communication afin d'influencer les anticipations des agents dans un contexte de taux d'intérêt très bas. Ainsi, les banques centrales ont mis en œuvre des politiques monétaires non-conventionnelles dont l'objectif fondamental a été d'ancrer les anticipations des participants au marché en période de crise. Dans les deux cas, la politique informationnelle du régulateur joue un rôle central et répond de manière stratégique à la réaction du marché. Enfin, la quatrième section conclut en évoquant plus précisément le jeu stratégique informationnel entre marché et régulateur.

\section{2 - Marché et information}

Cette section analyse les difficultés du marché à intégrer l'information pertinente au moyen de trois questions. Premièrement, pourquoi dans certains contextes, les investisseurs ne tiennent-ils pas compte des informations / indicateurs de crise? Nous verrons ainsi qu'en situation d'incertitude, les participants au marché peuvent sous-estimer la probabilité d'occurrence de résultats défavorables (si de tels résultats sont apparus dans un passé relativement lointain): c'est l'hypothèse de myopie au désastre. Cette hypothèse nous permet d'illustrer comment la bulle s'est construite sur le marché des subprimes. Deuxièmement, pourquoi les agents ne sont-ils pas incités à rechercher l'information pertinente? Il existe un courant de la littérature qui explique la crise financière par une insuffisante production d'information de la part des agents privés car celle-ci serait sous-optimale. Une telle insuffisante production d'information pourrait aussi expliquer la 
phase de boom. Troisièmement, pourquoi les investisseurs peuvent-ils sur-réagir à certaines informations? Dans certaines circonstances, la connaissance commune ${ }^{2}$ de certaines variables économiques peut conduire les participants au marché à leur accorder trop d'importance par rapport à leur contenu informationnel. L'analyse sous forme de jeu de concours de beauté keynésien permet de rendre compte de l'impact que peut avoir la révélation publique de certains indicateurs en termes de sur-réaction du marché et de crise.

\section{Pourquoi les investisseurs ne tiennent-ils pas compte des informations?}

L'hypothèse de myopie au désastre - avancée initialement par Guttentag et Herring [1986] permet d'apporter des éléments de réponses à cette question. Cette hypothèse cherche à expliquer pourquoi les crises financières sont des événements récurrents. A cette fin, Guttentag et Herring analysent le processus de décision des investisseurs en situation d'incertitude. La myopie au désastre est une situation dans laquelle les agents tendent à sous-estimer la probabilité de résultats défavorables. Un tel comportement est particulièrement probable pour des événements à faible probabilité apparus dans un passé relativement lointain. Guttentag et Herring utilisent les outils de la psychologie cognitive pour construire leur raisonnement. D'après cette approche, les agents économiques basent leurs règles de décisions sur une heuristique simple largement fondée sur des régularités empiriques. ${ }^{3}$

Dans un environnement incertain, les individus utilisent des probabilités subjectives. Une probabilité subjective est un jugement personnel concernant la probabilité d'occurrence d'un événement particulier. Les individus n'utilisent pas un calcul précis, mais fondent leur jugement sur une évaluation raisonnable. La probabilité subjective d'un individu à propos d'un événement décrit son degré de croyance à propos de l'occurrence de cet événement. Il n'y a pas de calcul formel. Les expériences passées sont très importantes pour former de telles probabilités. Au contraire, les probabilités objectives estiment l'occurrence d'un événement en se fondant sur l'observation réelle, plutôt qu'une estimation subjective.

D'une part, l'heuristique de perception («availability heuristic ») - qui se réfère à la facilité avec laquelle le preneur de décision peut imaginer qu'un événement se produira - repose sur le fait que plus la période depuis laquelle un événement s'est produit est grande, plus la probabilité subjective accordée à cet événement est faible. D'autre part, en-dessous d'un certain seuil, cette probabilité subjective est nulle. Ce niveau est appelé heuristique de seuil («threshold heuristic»). Ce seuil exacerbe le biais de sous-estimation de la probabilité du choc. Plus précisément, il s'agit de la règle empirique avec laquelle les preneurs de décision allouent leur rare temps disponible pour analyser ces probabilités.

La facilité avec laquelle le preneur de décision peut imaginer qu'un événement va se produire dépend de la période de temps qui s'écoule avant l'occurrence d'un événement : plus cette période est longue, plus la probabilité subjective attribuée à cet événement sera faible (Graphique 1). En- 
dessous d'un certain seuil, cette probabilité subjective est de zéro, ce qui exacerbe le biais de sousestimation de probabilité d'un choc.

Graphique 1 L'hypothèse de myopie au désastre



Source : les auteurs, à partir de Herring [1998].

L'hypothèse de myopie au désastre fait écho au modèle macroéconomique de Minsky [1977] fondé sur le paradoxe de la tranquillité selon lequel les crises financières résultent d'excès financiers pendant la période de croissance car les investisseurs, les entreprises et les ménages tendent à devenir de plus en plus optimistes. Williams [2012] modélise la théorie de Minsky. Il présente un modèle dynamique de booms et busts dans le prix des actions. Les individus ont le choix entre investir dans des titres qui procurent un rendement certain et constant à chaque période et des actions qui procurent un rendement incertain. Dans les rendements des actions, il y a une part exogène et une part endogène (liée aux prix). Williams résout d'abord le modèle dans le cas d'anticipations rationnelles: les agents connaissent la vraie moyenne et la vraie volatilité des dividendes et donc le prix d'équilibre sur le marché des actions (et la part de leur portefeuille investie dans les actions) est constant à chaque période. Les rendements sont ainsi identiquement distribués à chaque période. Il considère ensuite le cas où les agents ont des croyances fixes au fil des périodes mais arbitraires. Dans ce cas, les valeurs d'équilibre convergent vers celles de l'équilibre en anticipations rationnelles si et seulement si les paramètres estimés par les agents sont les vrais paramètres. Ensuite, il s'intéresse au cas où les croyances des agents ne sont plus fixes : les agents actualisent leurs croyances au fil des périodes. Il envisage alors ce qui se passe quand les agents actualisent leurs croyances suivant deux règles d'actualisation différentes : la première, du « decreasing gain learning» (impliquant que les agents attribuent le même poids à chacune des 
observations dans le temps, aussi anciennes soient-elles) et la deuxième, du «constant gain learning » (impliquant que les agents attribuent davantage de poids aux données plus récentes). Dans ces deux cas, les prix et la part du portefeuille de l'agent représentatif en actions ne sont plus constants car les paramètres estimés ne le sont plus au fil des périodes. S'il y a une succession de chocs positifs sur les dividendes, les agents croient que les paramètres ont changé (que la moyenne a augmenté et la volatilité a baissé) et donc la demande pour les actions augmente. Par conséquent, les prix augmentent et les rendements réalisés augmentent alors aussi, ce qui entretient le processus, jusqu'à ce qu'il y ait une succession de chocs négatifs. Puisque les périodes précédentes ont conduit les agents à investir une part importante de leur richesse post-consommation en actions, de petites variations dans leur portefeuille entraînent de grandes variations des prix, et le processus se perpétue dans l'autre sens.

Dans la lignée de la littérature sur le boom du crédit, Boz et Mendoza [2013] proposent un modèle théorique cherchant à rendre compte de la croissance des crédits accordés aux ménages et, plus largement, de l'accroissement du levier d'endettement. Ce modèle peut être relié à l'aveuglement au désastre et à la théorie de Minsky. Le point de départ de leur analyse consiste à considérer que ces évolutions sont liées à deux mouvements principaux. D'une part, l'introduction d'innovations financières à travers l'émission de nouveaux instruments financiers générant des flux financiers à travers une large variété d'actifs. Il s'agit de la titrisation. D'autre part, des réformes financières qui ont modifié de manière radicale le cadre légal et réglementaire des marchés financiers. Le modèle montre que l'innovation financière peut conduire à une sous-estimation du prix du risque associé à ce nouvel environnement financier. A son tour, cette sous-estimation peut induire une croissance forte et soudaine du crédit et des prix d'actifs suivie d'un effondrement. La sous-estimation du risque repose sur le fait que les agents manquent de données sur les défauts de paiements et les performances de ces nouveaux instruments financiers. La croyance dans la diversification des risques conduit les agents à considérer que les actifs issus de ces innovations sont sans risque. Cette idée fait écho à l'hypothèse de myopie au désastre, en ce sens que le modèle repose sur l'illusion qu'il existe très peu de risque, en partie parce que les agents ne peuvent pas bien l'estimer à cause des innovations.

Plus précisément, le modèle de Boz et Mendoza [2013] se focalise sur le rôle de l'innovation financière dans un environnement caractérisé par de l'information imparfaite et par des imperfections sur les marchés du crédit. Les agents découvrent le véritable degré du risque du nouvel environnement financier avec le temps. L'apprentissage est de type bayésien. A long terme, les croyances convergent vers le vrai régime de changements de probabilités (true regimeswitching probabilities) qui correspond à la solution en anticipations rationnelles. Le processus d'apprentissage interagit avec la contrainte sur le collatéral qui spécifie que la dette des agents privés ne peut pas excéder une fraction de la valeur de marché des actifs qu'ils détiennent. Les innovations financières sont modélisées comme un changement structurel augmentant la limite du 
levier d'endettement. Ainsi, les innovations financières font passer l'économie à un état de levier élevé. La contrainte sur le collatéral permet de générer dans le modèle un mécanisme du type amplification financière. Les auteurs mettent en interaction cette contrainte avec les croyances des agents à propos du degré de risque des innovations financières. Plus précisément, le modèle engendre un cycle du type boom - bust dans la mesure où la sous- (sur-) estimation du risque conduit à une sur- (sous-) estimation de la valeur des collatéraux, de type Minsky. Un choc négatif sur le collatéral fait brusquement passer l'économie d'un état avec levier élevé à un état où le levier est bas.

Une implication importante du modèle de Boz et Mendoza [2013] est que les superviseurs doivent surveiller les institutions financières particulièrement dans les périodes d'innovations et de réformes financières. En effet, dans ces périodes, les agents ne connaissent pas, par définition, la vraie nature du risque lié à ce nouvel environnement.

L'hypothèse de myopie au désastre souligne le fait que dans des circonstances très optimistes, les investisseurs ignorent l'information pertinente concernant un degré de risque croissant. Une telle explication est particulièrement pertinente pour comprendre la crise financière récente. Le graphique 2 montre différents indicateurs relatifs à l'évolution du marché immobilier américain depuis le début des années 2000.

Graphique 2 Le secteur immobilier américain

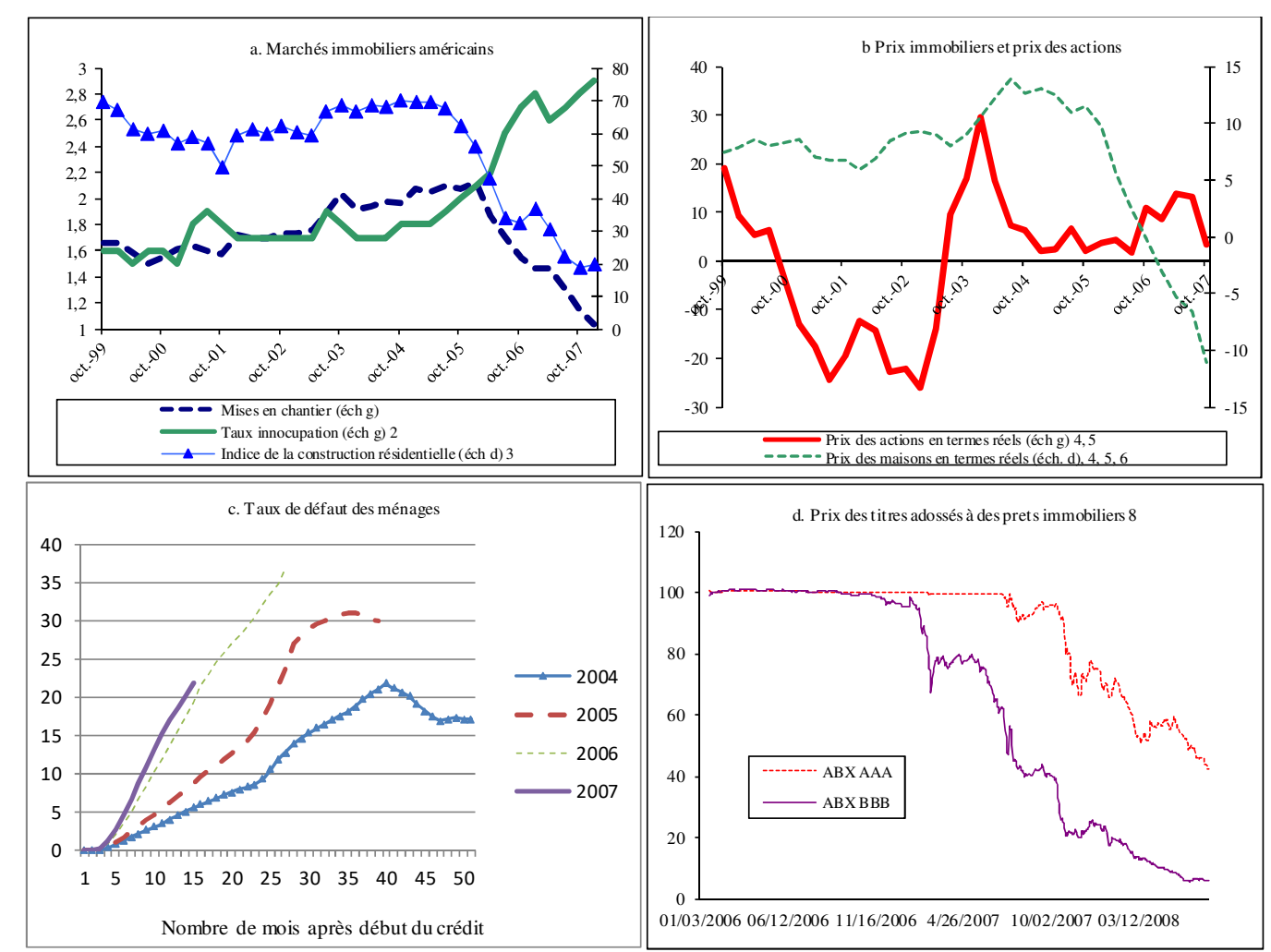

1 En millions ; données cvs annualisées. 2 Proportion de logements vacants à vendre dans le parc immobilierrésidentiel; en \%.

3 Mesure de la vigueur du marché des maisons individuelles ; une valeur supérieure à50 dénote une majorité de réactions positives.

4 Variation sur 4 trimestres, en \%. 5 Corrigé des prix à laconsommation. 6 Indice S\&P/Case-Shiller des prix de l'immobilier.

$760+$ jour du défaut, en \% de la valeur initiale. 8 ABX: indice des credit default swaps sur les titres adossés à des crédits immobiliers

Sources : BRI [2008] : 15 et FMI [2008] : p. 6-7. 
La caractéristique principale de ce graphique est que la détérioration du marché immobilier américain a commencé environ au milieu de l'année 2005 avec le déclin de l'indice de construction (panel a) et des prix immobiliers (panel b), et l'accroissement du taux de vacance (panel a). D'après le panel c, l'accroissement des défauts sur le marché subprimes suggère une détérioration significative du marché du crédit hypothécaire américain. Le point important est le suivant : alors que toutes les données montrent que l'explosion immobilière se termine en 2005 aux Etats-Unis, les prix se référant aux obligations adossées à des prêts hypothécaires ne changent pas avant mi2007 (panel d).

Pourquoi les investisseurs ne tiennent-ils pas compte de cette information? Il semble que le motif principal soit que - dans la mesure où les prix immobiliers américains ont augmenté sans interruption depuis 1995 - les investisseurs aient cru qu'un retournement permanent du marché immobilier américain était improbable. De ce fait, ils ont ignoré l'évolution des prix immobiliers, même si leur tendance paraissait clairement insoutenable à long terme. En effet, comme l'ont souligné Reinhart et Rogoff ([2009] : p. 207), l'augmentation cumulée des prix immobiliers a atteint $92 \%$ entre 1996 et 2006, soit plus de trois fois l'accroissement observé entre 1890 et 1996. Même s'il est difficile de déterminer avec exactitude le moment du principal choc, la décision de la banque française BNP Paribas le 9 août 2007 de suspendre de manière temporaire le calcul de la valeur liquidative ainsi que des abonnements ou des rachats de deux fonds spécialisés sur le marché des subprimes, a représenté un signal majeur quant à l'ampleur de la détérioration de ce marché. Après l'occurrence de ce choc, les anticipations des investisseurs ont soudainement changé : leur appétit pour/goût du risque s'est considérablement effondré. Sur le graphique 3, le panel a montre cette relation. L'indice VIX (fourni par le Chicago Board Options Exchange Market Volatility Index) mesure les anticipations du marché de la volatilité à court terme véhiculée par l'indice des prix d'options d'achat d'actions. Dans la mesure où la volatilité est généralement un bon indicateur de turbulence financière, l'indice VIX est considéré comme une jauge de la peur des investisseurs. Le VIX tend à être élevé pendant les périodes de turbulences financières et de forte aversion au risque de la part des investisseurs. Pendant les périodes calmes, le niveau du VIX tend à diminuer. Cet indice est donc un indicateur de la perception du risque par les investisseurs. Comme le montre le graphique 3, il n'y a pas eu de changement majeur dans le comportement du VIX - en dépit de la détérioration dans le secteur immobilier américain - jusqu'à l'annonce faite par BNP Paribas. La réaction des investisseurs a été soudaine. Du point de vue de l'attitude face au risque, les investisseurs ont fait une «indigestion » : ils ont voulu complètement éviter tous les actifs risqués. Ainsi, les investisseurs n'ont accepté de ne détenir que des actifs sans risque, c'est-à-dire des bons du Trésor américain dont les revenus se sont effondrés après le 9 août 2007 (voir graphique 3, panel b pour ceux de maturité 1 mois). Cet effet est appelé la fuite vers la qualité (flight to quality). 
Graphique 3 Aversion au risque des investisseurs et fuite vers la qualité. Données journalières de janvier 2004 à décembre 2007

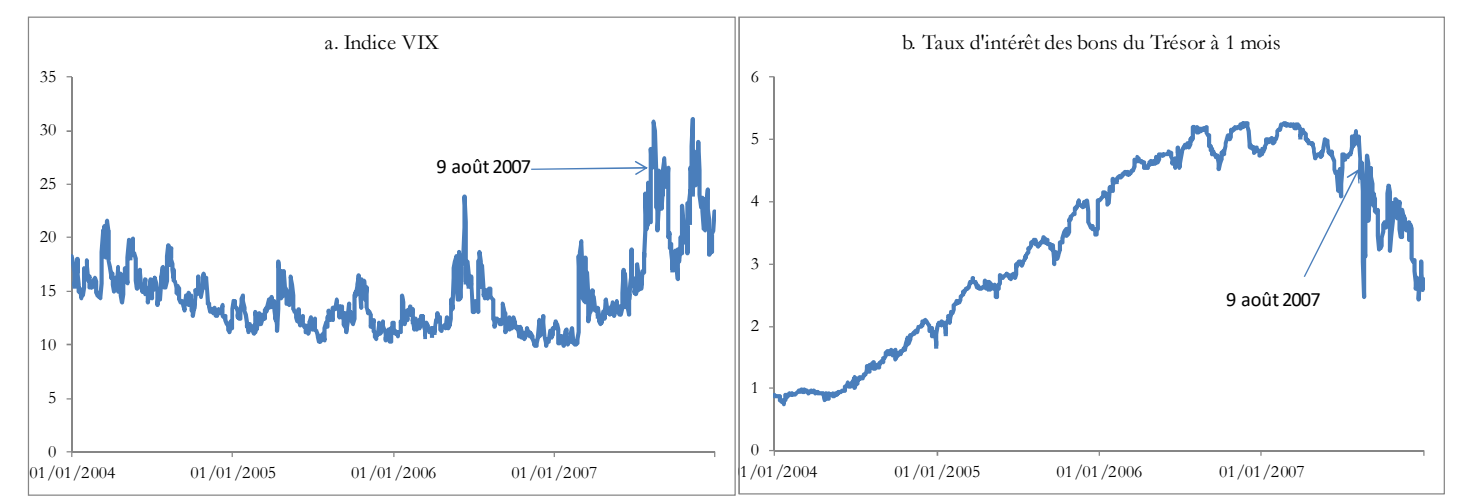

Sources : Macrobond.

Cornand et Gimet [2012] ont cherché à voir dans quelle mesure les banques ont fait preuve d'un aveuglement au désastre au cours de la crise des subprimes. Elles considèrent à cette fin une approche dynamique de la prise de risque appréhendée à travers le degré de déséquilibre de maturité dans les bilans bancaires. A partir de la méthode des moments généralisés, les auteurs montrent que la prise de risque à la période $t$ est influencée par la prise de risque à la période précédente $(t-1)$, ce qui suggère la présence d'un aveuglement au désastre. Les auteurs montrent que 2007 marque une rupture dans la prise de risque, ce qui est là encore conforme à l'hypothèse de l'aveuglement au désastre : un choc négatif majeur tend à réduire brutalement la prise de risque. Haldane [2009] souligne que le secteur privé mais aussi le secteur public souffrent de myopie au désastre. Plus précisément, l'évaluation du degré de risque - la value at risk pour le secteur privé et les tests de stress (stress-tests) ${ }^{4}$ pour le secteur public - était fondée sur des données et des hypothèses relatives à l'âge d'or («Golden Era »), c'est-à-dire la période de 1998 à 2007. Cette période a été caractérisée par une volatilité historiquement faible générant un faible niveau de risque ayant pu conduire à la myopie au désastre. En utilisant des données anglaises - remontant parfois jusqu'au $17^{\text {m̀me }}$ siècle - Haldane [2009] montre que les faibles niveaux de volatilité macroéconomique et financière pendant l'âge d'or ont été sans précédent. Cette absence de perspective historique pour évaluer le niveau de risque a conduit à la myopie au désastre.

\section{Pourquoi les investisseurs ne sont-ils pas incités à rechercher l'information ?}

Un certain nombre de modèles ont proposé une interprétation de la crise financière internationale en termes d'insuffisante production d'information par les agents privés. Le point important de ces modèles est de suggérer que si, en l'absence de crise, il est optimal pour les agents privés de ne pas produire de l'information, ce comportement devient sous-optimal en période d'instabilité. Cette insuffisante production d'information est sous-optimale au niveau social.

Hanson et Sunderam [2013] analysent un environnement économique dans lequel l'information est coûteuse à acquérir. Dans cette perspective, l'émission de produits structurés à travers la titrisation 
- fondée sur le regroupement (la mise en pool) de crédits selon un découpage en tranches contenant chacune des crédits classés selon divers niveaux de risques - est interprétée comme une opération permettant de réduire les coûts de la production d'information. Plus précisément, en l'absence de titrisation, les investisseurs seraient amenés à produire de l'information concernant les actifs émis. Or, d'un point de vue économique, une telle production d'information peut être inefficiente en raison des coûts associés à sa duplication. Avec la structuration des actifs en tranches, les flux de liquidité liés aux actifs émis sont associés à des titres senior et junior, ce qui tend à minimiser les inefficiences liées à la duplication. Les titres seniors étant considérés comme très faiblement risqués, ils se caractérisent par une absence de sensibilité à la production d'information (informationally insensitive). Les titres junior sont quant à eux sensibles aux informations, mais ils sont détenus par des investisseurs spécialisés. L'information sur les actifs titrisés n'est pas inexistante, mais elle est coûteuse à produire dans la mesure où les investisseurs doivent non seulement disposer de données historiques sur la performance des prêts, mais aussi de modèles permettant de simuler les flux de liquidité liés aux actifs et d'analyses très spécialisés. Cette infrastructure est d'autant plus coûteuse (en termes de coûts d'opportunité) que la titrisation permet d'obtenir des titres non sensibles à l'information.

A partir de ce cadre d'analyse, Hanson et Sunderam [2013] cherchent à montrer que si, en période normale, cette économie dans la production d'information est efficace, elle peut devenir problématique dans les états défavorables de l'économie. En effet, dans ces derniers, l'absence d'information peut exacerber la sélection adverse - au sens où les problèmes d'insuffisance d'information pour distinguer entre emprunteurs de bonne et de mauvaises qualités deviennent plus importants - et par là-même favoriser un effondrement des marchés. Le modèle engendre ainsi la situation suivante : dans les périodes normales (les bons états), les agents produisent de manière optimale un montant excessif de titres non sensibles à l'information. De manière endogène, les investissements réalisés dans l'infrastructure productrice d'information sont trop faibles. Il en résulte alors un effondrement des marchés dans les mauvais états. Afin de comprendre la dynamique du modèle conduisant à ce résultat, il convient d'introduire une distinction entre les investisseurs ayant investi dans l'infrastructure d'information (appelés investisseurs informés) et ceux qui n'ont pas investi (investisseurs non informés). Sur le marché de l'occasion, ces derniers sont confrontés à un phénomène de sélection adverse quand ils échangent avec les investisseurs informés. A travers l'émission de titres risqués sensibles à l'information, les originateurs exercent une influence sur les profits des investisseurs informés. En effet, plus la proportion de titres risqués à l'émission est élevée, plus les investisseurs informés peuvent tirer profit de leur investissement préalable dans des infrastructures productrices d'information. Dans les états normaux, comme les différences dans les gains entre investisseurs informés et non informés sont relativement faibles, la sélection adverse n'est pas intense. Ainsi, les originateurs peuvent financer leurs pools de prêts en émettant des titres peu sensibles aux informations. Ce comportement a une conséquence 
importante : les investisseurs informés n'ont qu'un faible avantage pour capter des profits. Dès lors, les investisseurs ne sont pas incités à rechercher de l'information. En conséquence, ex ante, l'investissement dans des infrastructures productrices d'information est insuffisant. Dans les états défavorables, les gains liés aux pools de mauvaise qualité diminuent et l'ampleur du phénomène de sélection adverse augmente. En conséquence, les fonds que peuvent lever les émetteurs de produits titrisés auprès des investisseurs non informés diminuent. Les investisseurs informés sont quant à eux moins sensibles à la sélection adverse. Ils représentent donc une source relativement sûre de capital dans les états défavorables, mais ils sont peu nombreux. Ainsi, dans les états défavorables, le montant des fonds que peuvent lever les originateurs diminue, ce qui peut engendrer un effondrement du marché de la titrisation. Le graphique 4 relatif aux émissions de titres adossés à des actifs aux Etats-Unis (asset-backed securities) illustre ce processus qui se produit brutalement en 2007.

Graphique 4 Etats-Unis : émissions de titres adossés à des actifs, en millions de dollars

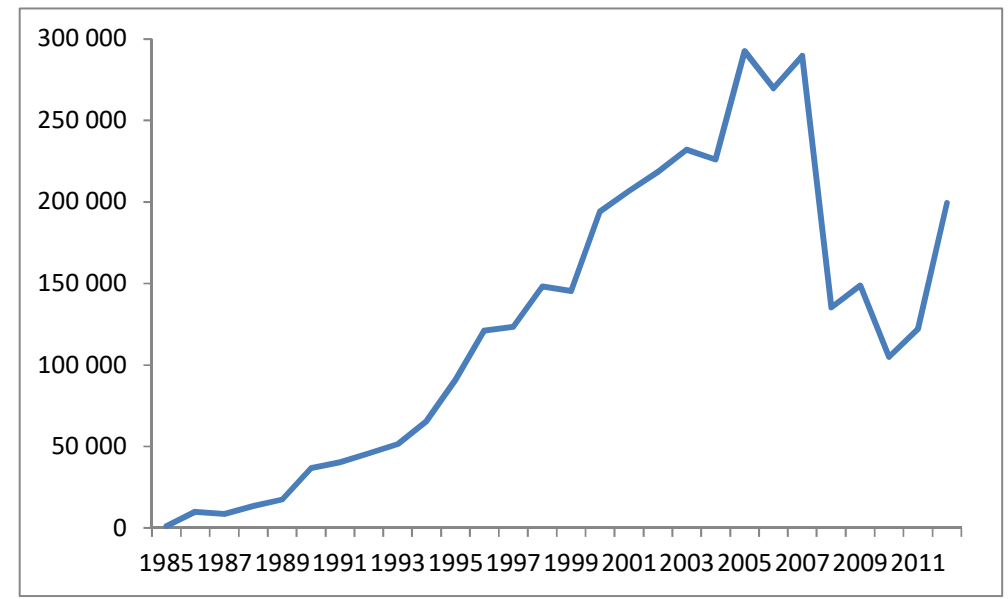

Source : Securities Industry and Financial Markets Association (SIFMA)

Ce recul très important des émissions doit être rapproché de la baisse des prix de ces actifs (graphique 2, panel d).

Le point important du modèle de Hanson et Sunderam [2013] est que les rendements privés liés à l'investissement dans l'infrastructure d'information peuvent être inférieurs au rendement social. En effet, selon les auteurs, le marché n'est pas en mesure de faire face à la friction financière liée à la sélection adverse pour deux raisons principales. La première est le problème de l'engagement qui repose sur le fait que les originateurs, dans les bons états, ne peuvent pas s'engager à limiter le recours aux émissions de titres peu sensibles à l'information. Avant que le bon ou le mauvais état ne se réalise, les originateurs peuvent vouloir s'engager ex ante à émettre des titres sensibles à l'information, ce qui peut encourager les investisseurs à rechercher de l'information. Cependant, une fois le bon état réalisé, ils maximisent leur profit en émettant des titres peu sensibles à l'information. Les investisseurs anticipent ce comportement et n'investissent donc pas dans la recherche d'information. La seconde raison est liée à la présence d'externalités. Un originateur 
individuel peut émettre des titres sensibles à l'information dans les bons états et conduire les investisseurs à rechercher de l'information. Cependant, cet originateur n'est pas certain de recevoir des fonds de la part de ces investisseurs informés dans les mauvais états. L'infrastructure informationnelle est en fait un bien public du point de vue des originateurs. Les coûts en période normale sont diffus mais les bénéfices sont quant à eux concentrés dans les mauvais états. Dès lors, il est optimal pour les originateurs d'éviter le coût plus élevé de l'émission de titres sensibles à l'information dans les périodes normales. La principale implication du modèle est alors de réguler les décisions des originateurs en termes de structure du capital en les incitant à augmenter les émissions de titres risqués et sensibles à l'information, permettant ainsi aux investisseurs informés d'obtenir des profits dans les bons états. Les investisseurs seront ainsi incités à investir dans l'infrastructure informationnelle, ce qui limitera les inefficiences liées à la sélection adverse dans les mauvais états.

Considérant que l'effondrement des marchés de produits structurés a été interprété comme la conséquence du fait que les agents ont manqué d'information pour déterminer leurs prix et ainsi les échanger, Pagano et Volpin [2012] cherchent à expliquer pourquoi leurs émetteurs ont choisi l'opacité plutôt que la transparence. En effet, les émetteurs devraient anticiper que les marchés de l'occasion peuvent devenir illiquides du fait de ce manque de transparence. Dès lors, il convient de comprendre pourquoi les émetteurs et les investisseurs peuvent avoir intérêt à échanger des produits structurés sur lesquels ils n'ont que peu d'information. L'explication repose sur la divergence entre les bénéfices privés et sociaux d'une transparence accrue des actifs titrisés.

Selon Pagano et Volpin [2012], les émetteurs sont incités à mettre sur le marché des titres ayant peu de contenu informationnel afin d'augmenter la liquidité du marché primaire. En effet, une information détaillée sur les produits structurés est coûteuse à acquérir et demande une expertise avancée pour l'interpréter correctement. En conséquence, il existe potentiellement peu d'investisseurs suffisamment sophistiqués capables de comprendre les implications en termes de formation des prix d'une information complexe. En supposant que des titres ayant un contenu informationnel complexe soient émis, les émetteurs pourraient voir la liquidité du marché primaire diminuer en raison du fait que les investisseurs non sophistiqués seraient confrontés au problème de la «malédiction du vainqueur ». Selon cette dernière, les investisseurs non informés et informés étant en concurrence sur les marchés pour acquérir des titres, les premiers tendent à surévaluer la valeur des titres émis relativement aux investisseurs sophistiqués, ce qui ne les incitent pas à venir sur le marché primaire. Cet accroissement de la liquidité sur le marché primaire peut réduire la liquidité sur le marché secondaire causant ainsi une baisse des prix. La raison en est que l'information non divulguée au moment de l'émission peut être découverte/apprise par les investisseurs sophistiqués plus tard. Autrement dit, les investisseurs sophistiqués cherchent à acquérir davantage d'information sur le marché secondaire, ce qui conduit la sélection adverse à 
passer du marché primaire au marché secondaire. Les émetteurs de titres sont dès lors confrontés à un arbitrage entre liquidité du marché primaire et liquidité du marché secondaire.

Pagano et Vopin [2012] suggèrent que les émetteurs ne choisissent jamais de divulguer une information précise au moment de l'émission même s'ils anticipent une liquidité inférieure sur le marché secondaire. En effet, si les émetteurs choisissent la transparence, ils supportent les conséquences négatives de la sélection adverse sur le marché primaire alors que dans un régime d'opacité, le coût lié à la sélection adverse n'apparait que si les investisseurs sont frappés par un choc de liquidité. Or, la probabilité qu'un tel choc se réalise est inférieure à un. Les émetteurs n'ont donc pas d'incitation privée à émettre des titres contenant de l'information complexe. Lorsque l'illiquidité du marché secondaire provoque un processus cumulatif de défauts et des ventes de détresse, la liquidité du marché secondaire a une valeur sociale qui excède sa valeur privée. On en déduit alors que le degré de transparence choisi par les émetteurs est inférieur à celui socialement optimal.

La principale implication du modèle repose sur la régulation de la production d'information sur le marché primaire. Les auteurs soulignent ainsi que le régulateur pourrait demander aux émetteurs de produits structurés de fournir davantage de données détaillées concernant les prêts sous-jacents aux pools ou aux agences de rating de fournir des notations plus sophistiquées (prenant par exemple en compte la probabilité de défaut mais aussi la corrélation du risque spécifique à un titre avec le risque systématique).

Le levier d'endettement des agents non financiers et des banques a souvent été mis en avant pour expliquer pourquoi un choc sur un marché de taille limitée - le marché des crédits hypothécaires américains à risque - avait engendré une crise financière d'une ampleur aussi considérable. L'accent mis sur le levier repose en partie sur le fait que cette crise financière a été précédée par un boom du crédit. Le modèle de Gorton et Ordonez [2012] s'inscrit dans cette littérature. Les auteurs cherchent à identifier les facteurs permettant de comprendre pourquoi le levier d'endettement peut avoir un tel potentiel d'amplification des chocs. A cette fin, ils élaborent une théorie des crises financières fondée sur la dynamique de la production et de l'évolution de l'information sur les marchés de dette à court terme faisant l'objet d'un collatéral. Cette dernière inclut les engagements bancaires et les instruments du marché monétaire. Elle apporte aux agents économiques des services de transaction en permettant des échanges sans crainte de sélection adverse. Le graphique 5 montre l'expansion des prises en pension (les repurchase agreements) aux Etats-Unis depuis le milieu des années 90 et leur recul marqué à la suite de la crise financière. 
Graphique 5 Etats-Unis : prises en pension, engagements nets, en millions de dollars

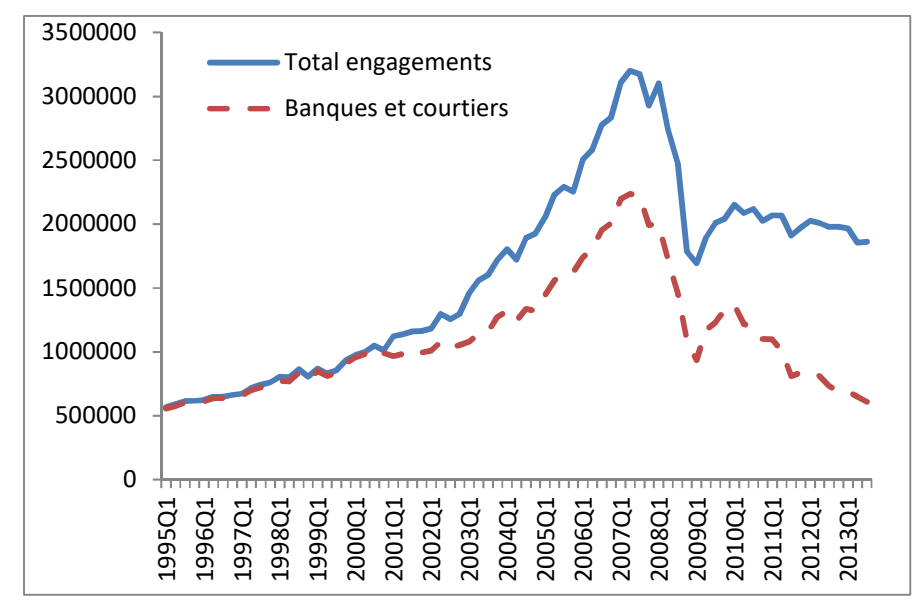

Source : Federal Reserve Bank, Flows of Funds.

Selon Gorton et Ordonez [2012], la caractéristique fondamentale de ces dettes à court terme est de ne pas être sensible à l'information (information-insensitive). Dès lors, il n'est pas profitable pour un agent de produire de l'information privée concernant le collatéral adossé à cette dette. Or, dans les périodes de crise financière, il apparaît une perte de confiance soudaine dans la qualité de la dette à court terme. Celle-ci devenant sensible à l'information, les agents doivent produire de l'information pour déterminer la qualité du collatéral. Cependant, l'information étant coûteuse à produire, les agents économiques ne sont pas incités à produire de l'information dans tous les états de l'économie. Gorton et Ordonez [2012] font l'hypothèse que le collatéral est sujet à deux types de chocs. D'une part, les chocs idiosyncratiques qui se caractérisent par le fait qu'au cours du temps, sans production d'information, la valeur perçue de tout collatéral tend à être la même dans la mesure où il apparait un retour vers la qualité moyenne perçue le marché. D'autre part, les chocs agrégés qui peuvent faire baisser la valeur des collatéraux et induire une crise de confiance sur les marchés de dette à court terme.

Dans la période précédant la crise financière, on observe un boom du crédit qui repose sur le fait que des entreprises sont en mesure d'émettre de la dette avec des collatéraux dont la qualité est surestimée puisqu'assimilée à la qualité moyenne des collatéraux. Autrement dit, des firmes peuvent accroître leur levier d'endettement relativement à la situation qui aurait prévalu si les agents avaient produit de l'information précise sur la qualité de chaque collatéral. La conséquence est qu'un choc négatif agrégé faisant baisser la valeur des collatéraux aura alors des implications macroéconomiques plus importantes que le même choc dans un environnement informationnel marqué par la connaissance de la qualité des collatéraux. L'émission de produits structurés amplifie ce phénomène puisque des collatéraux de bonne qualité sont mélangés avec des collatéraux de mauvaise qualité. L'économie connait une situation de fragilité croissante liée au fait que les agents n'intègrent pas les conséquences futures de la faible production d'information aujourd'hui. 


\section{Pourquoi les agents sur-réagissent-ils à l'information ?}

Dans un contexte de concours de beauté keynésien ${ }^{5}$, les investisseurs peuvent sur-réagir aux informations publiques. Morris et Shin [2002] montrent qu'accroître la transparence risque d'augmenter l'instabilité financière du fait de la différence de nature entre informations publique et privée. Le point important est que l'information publique (et tout particulièrement les annonces des banques centrales) présente des attributs qui en font un instrument à double tranchant. D'une part, elle donne de l'information sur les fondamentaux de l'économie, mais, d'autre part, elle sert également de point focal aux croyances du groupe dans son ensemble et peut coordonner les actions des agents. Contrairement à l'information privée, l'information publique permet à un investisseur de faire des hypothèses sur les actions des autres participants. Cette caractéristique provient du fait que l'information publique fournit de l'information sur ce que les autres participants ont observé. Elle permet donc de déduire ce que les autres participants vont jouer. En d'autres termes, si l'information privée ne fait que donner une indication sur l'état de l'économie, l'information publique, elle, remplit les deux rôles. Dans ce cadre, Morris et Shin montrent que les agents peuvent sur-réagir aux annonces. Cela signifie qu'ils peuvent porter une attention trop grande aux nouvelles annoncées publiquement, et si ces nouvelles manquent de précision (si elles sont bruitées), les agents peuvent être amenés à prendre des décisions non justifiées par les fondamentaux. Dans un tel contexte, la valeur sociale de l'information publique est ambiguë.

L'analyse de Gorton [2008, 2009a, b] sur l'absence de connaissance commune et l'effet de la révélation de signaux publics générant une sur-réaction sur le marché va dans le même sens. En effet, la crise des subprimes est caractérisée par l'existence d'une forte complexité des actifs financiers. Comme nous l'avons rappelé précédemment, une partie des crédits subprimes a été transformée en obligations par le processus de titrisation. Ces obligations ont ensuite été subdivisées en tranches pour disperser le risque. En conséquence, l'origine des produits structurés devient très diffuse. La plupart des titres adossés à des actifs (asset-backed securities) sont ainsi des produits complexes contenant des dizaines ou des centaines d'hypothèques sous-jacentes. La documentation les concernant peut comporter une centaine de pages. Ainsi est-il extrêmement difficile pour un investisseur individuel de surveiller l'évolution de l'exposition au risque (Bean [2009]).

Gorton souligne qu'une telle complexité a créé une illusion de transparence, une situation d'opacité telle qu'ex ante (avant la crise) il y avait absence de connaissance commune sur les difficultés financières touchant notamment le marché des subprimes. Dans ce contexte, l'annonce des difficultés économiques a pu déclencher la crise. Lorsque le nombre de défauts sur certains prêts hypothécaires à risque aux États-Unis mis en place en 2006 et 2007 a commencé à être beaucoup plus élevé que prévu, il y a eu une prise de conscience du fait que les pertes pourraient être beaucoup plus importantes sur certains de ces titres qu'on ne le croyait auparavant. Les croyances des investisseurs ont connu un changement radical. Plus précisément, alors que les investisseurs 
avaient pu penser initialement que les rendements se comporteraient selon une distribution traditionnelle, ils ont ensuite considéré que les rendements pouvaient suivre une distribution inhabituelle conduisant à un état du type incertitude knightienne (Caballero et Krishnamurthy [2008]). Un autre exemple emblématique est celui du gel de ses fonds par BNP Paribas le 9 août 2007 : il a en effet révélé au marché à quel point le marché des subprimes était un marché risqué.

Bacchetta et al. [2010] soulignent que des événements comme l'effondrement de Lehman Brothers peuvent représenter des points focaux pour les investisseurs, changeant la façon dont les fondamentaux sont interprétés et changeant par là même la perception du risque par les investisseurs. Ceci peut conduire à des paniques, des pics dans l'émergence de risques auto-réalisés et à un effondrement dans le prix des actifs. Bacchetta et al. [2012] expliquent de tels mouvements non associés à des changements dans les fondamentaux en mettant l'accent sur les changements dans la perception du risque. Ils observent que la baisse importante dans le prix des actifs a été associée avec des montées brutales des risques et par des mesures des risques devenant ellesmêmes plus volatiles dans les périodes de crises.

Au cours des dernières années, l'information disponible sur les produits et marchés liés au transfert des risques de crédit s'est considérablement accrue, et, plus précisément, les données sur les prix. Les indices et tranches d'indices donnent de l'information sur les prix au jour le jour à la fois pour les investisseurs et le public sur de nombreux marchés. D'après Gorton [2008 et 2009a], la publication d'indices $\mathrm{ABX}$ a créé de la connaissance commune sur le marché subprimes. Ainsi, chaque participant au marché peut connaître sa propre situation, mais également celle des autres. Les indices $\mathrm{ABX}$ sont des indices synthétiques du risque lié aux subprimes, créés en 2006 et qui représentent donc un signal public sur le marché. Avec la publication de ces indices, c'était la première fois que l'information sur la valeur et les risques associés aux subprimes était agrégée et révélée (bien que la localisation du risque et des pertes soit restée inconnue du fait de la complexité des interconnections). Ce signal public a clairement indiqué que la situation s'était détériorée et en 2007, l'indice ABX s'est effondré (voir graphique 2d).

Boyarchenko [2012] a modélisé la crise des subprimes en considérant les changements dans l'ambiguïté («ambiguity shifts»). Plus précisément, l'auteur décrit des situations dans lesquelles les investisseurs et les banques ne connaissent pas la vraie valeur de la distribution de probabilité de l'état de la nature et sa dynamique au cours du temps. Les agents doivent ainsi faire des estimations - en s'appuyant sur des données comptables et des données macroéconomiques disponibles publiquement - afin d'élaborer leur stratégie de prise de risque, mais ils n'accordent pas nécessairement un degré de confiance élevé dans ces estimations dans la mesure où ils ne sont pas certains d'utiliser le vrai modèle permettant de générer des données fiables. Dans ce contexte, les agents ont un biais pessimiste au sens où ils tendent à considérer que la probabilité de défaut à la période suivante est plus élevée que la probabilité qu'ils auraient pu estimer en l'absence d'ambiguïté («ambiguity-neutral »). Les signaux qu'ils reçoivent sont ainsi interprétés de manière 
pessimiste, donnant ainsi un poids très important aux nouvelles négatives. Boyarchenko [2012] montre que son modèle permet de comprendre les augmentations brutales des CDS des institutions financières après l'annonce de BNP Paribas en août 2007 ou les faillites de Bear Stearns et Lehman Brothers en $2008 .^{6}$

Cette situation de doute (pour une institution financière connaître sa propre situation, mais ne pas être certain si les autres sont solvables ou à risque) a également été observée sur le marché interbancaire où la liquidité s'est soudainement évaporée.

Un autre exemple est celui du rôle des agences de notation dans l'évaluation des produits financiers structurés (FMI, [2008]). Dans la deuxième moitié de 2007, les trois principales agences de notation ont été amenées à déclasser précipitamment un grand nombre de produits financiers structurés adossés aux crédits hypothécaires américains sur lesquels les taux de défaut avaient augmenté brusquement contrairement aux hypothèses émises auparavant. Parmi les titres déclassés, on a pu trouver des titres qui étaient notés AAA, note la meilleure possible correspondant à des actifs sans risque. Les notations de crédit ratings ont représenté un élément clé pour de nombreux investisseurs dans l'évaluation des produits de crédit structurés car ils ont été perçus comme fournissant une mesure du risque de crédit commune pour tous les instruments à revenu fixe.

\section{3 - Les réponses des autorités à la crise : quelles implications en termes de diffusion}

\section{d'information?}

En réponse à la crise, et plus particulièrement aux difficultés des marchés à intégrer l'information pertinente, les autorités de régulation et les banques centrales ont cherché à augmenter le flux d'information, la plus précise et complète possible et donc à accroitre la transparence.

En supposant que les régulateurs tendent à être davantage immunes à la myopie au désastre (Haldane, [2009]), ils doivent concevoir des scenarios de stress intégrant les événements extrêmes et les imposer aux institutions financières et examiner plus en profondeur les modèles utilisés par les institutions financières, en particulier les séries statistiques historiques utilisées pour estimer les risques. Nous examinons ainsi tout d'abord le lien entre information et gestion de l'instabilité bancaire, notamment via le rôle des tests de stress et dans quelle mesure ceux-ci permettent de répondre à la fois au manque d'incitation des participants au marché à rechercher l'information et à l'insuffisante attention des investisseurs aux informations diffusées.

Dans la mesure où la crise financière a un impact réel, les banques centrales ont répondu au choc par le biais de la politique monétaire. Aussi, de la même manière, afin de mieux gérer la réaction des marchés aux informations et à la mise en œuvre de la politique monétaire en situation de crise, les banques centrales ont été amenées à se tourner vers des politiques nouvelles, dites politique non-conventionnelles, dans lesquelles la communication au marché joue un rôle majeur. Il existe ainsi un jeu stratégique dynamique entre participants au marché et autorités monétaires, au sens où 
la banque centrale doit prendre en compte la réaction du marché à la mise en œuvre de sa politique monétaire et sa politique de communication.

\section{Gestion de l'instabilité bancaire et information}

A la suite de la crise de 2008, certaines banques centrales, notamment la Réserve Fédérale américaine aux Etats-Unis et la Banque Centrale Européenne (BCE) dans la zone euro, ont décidé de faire subir des tests de stress aux institutions financières pour évaluer leur capacité à résister aux chocs. En effet, la crise financière a soulevé la question du niveau de capitalisation des banques et en particulier des plus grandes institutions financières. ${ }^{7}$ Une question centrale pour les décideurs politiques et les banques et de savoir si les résultats de ces tests devraient être diffusés et, si tel est le cas, avec quel niveau de détail.

Les supervisory stress tests évaluent l'impact d'un scénario macroéconomique néfaste sur la rentabilité et la capitalisation d'un grand nombre de banques. Les superviseurs peuvent garder les résultats des tests confidentiels ou les rendre publics, réduisant ainsi l'opacité des banques ce qui permet aux investisseurs de mieux évaluer les forces et faiblesses des institutions financières et de restaurer la confiance. La motivation principale des tests de stress est liée au fait que l'opacité tend à augmenter en temps de crise (Flannery et al., [2013]) rendant nécessaire la recherche de moyens pour rassurer les marchés en période de difficultés : en diffusant de l'information sur les forces et les faiblesses des banques, les superviseurs visent à réduire l'incertitude sur le marché et prévenir les paniques. Les investisseurs qui bénéficieraient d'un large ensemble de données comparables d'une banque à l'autre et dans une certaine mesure certifiées par les superviseurs, pourraient mieux différencier les risques liés à telle ou telle institution. ${ }^{8}$

Diffuser ou non les résultats des tests de stress: les principaux enseignements de la littérature

Il existe une littérature récente qui évalue dans quelle mesure les résultats des tests de stress doivent être diffusés.

Les partisans de la diffusion des résultats des tests de stress font le lien entre sévérité de la crise financière récente et opacité bancaire. Ils soulignent le fait que de nombreuses banques ont pris des risques excessifs qui n'ont pas été révélés de sorte qu'ils n'ont pu être intégrés dans les prix fixés par le marché. La diffusion des résultats des tests de stress permettrait aux investisseurs de mieux comprendre les profils de risque de chaque institution, ce qui devrait contribuer à accrôtre la discipline de marché. Une telle discipline de marché, en retour, devrait éviter que les banques ne s'engagent ex ante à adopter des comportements trop risqués. Une plus grande transparence sur les risques bancaires pourrait aussi permettre au régulateur de mieux contrôler les banques et lui permettre d'intervenir suffisamment tôt par exemple pour recapitaliser les banques en difficulté. La diffusion des tests de stress devrait aussi permettre de restaurer la confiance des investisseurs dans le secteur bancaire et influencer à terme positivement l'économie réelle. L'effet des tests de stress sur l'opacité des banques et les primes de risque dépend du type de données qui est révélé au 
marché lorsque les résultats sont diffusés. Goldstein et Sapra [2012] passent en revue la littérature relative à la diffusion d'information concernant les tests de stress. Ils soulignent les avantages d'une telle diffusion. Ils suggèrent que la diffusion d'information détaillée est préférable à celle d'information agrégée car celle-ci permet aux participants au marché d'évaluer les causes de l'échec ou du succès au test.

Jusqu'à présent, nous avons essentiellement mentionné la littérature évoquant la possibilité de rassurer les marchés quant à la situation des institutions bancaires. Mais les tests de stress indiquant une situation moins bonne qu'anticipée pourrait également être bénéfique en termes de stabilité financière, par exemple parce que cela permet de prévenir la contagion (Shapiro et Skeie, [2012]).

Si les arguments en faveur de la diffusion des tests de stress semblent relativement intuitifs, les tests de stress ont également des conséquences (néfastes) inattendues. Ainsi, plutôt que de promouvoir la discipline de marché, s'ils ne sont pas formulés correctement, les résultats des tests de stress peuvent en réalité créer une panique, minant la confiance dans le secteur bancaire.

Das [2011] soutient l'idée selon laquelle l'annonce publique des résultats des tests de stress est imparfaite dans la mesure où les superviseurs ne peuvent pas réellement tester des scénarios suffisamment extrêmes (par exemple impliquant un défaut souverain) de peur d'effrayer les investisseurs. Si les scénarios de stress envisagés ne sont pas suffisamment extrêmes, les résultats de ces tests risquent tout simplement d'être ignorés par les marchés. De la même façon, si la situation économique suivant l'annonce des résultats des tests se détériore dans les mois qui suivent au-delà de ce qui avait été envisagé dans les tests de stress, les superviseurs peuvent perdre leur crédibilité conduisant à une forte incertitude sur le marché. C'est la raison pour laquelle certains superviseurs comme le CCAR (2012-11 US Comprehensive Capital Assessment Review) ont décidé de ne pas diffuser les résultats des tests. D'autres comme les 2011 European stress tests ont préféré diffuser davantage d'information qu'un seul signal binaire («réussit le test»; «échoue ») pour que les investisseurs puissent évaluer d'eux-mêmes la situation réelle des institutions financières. Cette littérature s'inscrit dans le débat sur la transparence en matière de supervision bancaire (GAO, [2010]).

Comme nous l'avons souligné dans la section 2, Morris et Shin [2002] montrent que diffuser de l'information peut être néfaste dans un contexte où les actions des agents sont des compléments stratégiques. Donner de l'information publique est dommageable dans la mesure où les agents attribuent un poids trop important à cette dernière car elle donne non seulement une indication sur l'état fondamental de l'économie, mais aussi de l'information sur ce que les autres pensent sur cet état de l'économie. Nous avons vu que ce type d'analyse permet d'expliquer en quoi le marché pouvait sur-réagir aux annonces publiques; ce même type d'analyse prévaut en matière de publication des tests de stress et peut être utilisé pour analyser cette fois-ci non pas directement la réaction du marché, mais ses conséquences pour le régulateur. Ainsi, Leitner [2012] montre qu'une diffusion trop importante d'information peut réduire la capacité du régulateur à extraire de 
l'information relative aux contrats complexes formulés par les banques les unes envers les autres ; de la même façon, Bond et Goldstein [2012] montrent que la diffusion d'information peut réduire la capacité du gouvernement à extraire de l'information sur le marché. En effet, si le marché surréagit aux annonces, il se produit une éviction de l'information privée. Le régulateur n'est ainsi plus à même, par son observation du marché, de déduire l'information qu'ont les investisseurs en propre.

Dans la même veine, selon Goldstein et Sapra [2012], dans un contexte avec friction (informationnelle) et compléments stratégiques entre les actions des participants au marché, les bénéfices associés à une meilleure discipline de marché peuvent être insuffisants pour justifier la diffusion d'information: les banques peuvent être amenées à faire de mauvais choix en matière d'allocation de portefeuille pour maximiser leurs chances de réussir les tests en abandonnant leur stratégie de long terme. Les traders peuvent attribuer un poids trop important à l'information publique relative aux tests de stress et être dés-incités à produire de l'information privée à propos des banques. Enfin, le contenu informationnel des prix de marché peut être affecté, rendant ces prix moins informatifs pour le régulateur/superviseur.

Bouvard et al. [2013] évaluent l'arbitrage lié à l'utilisation de tests de stress auquel sont confrontés les régulateurs pour établir le niveau optimal de transparence dans le système financier. Lorsque les institutions financières sont exposées à un risque de (non-renouvellement de) crédit (rollover risk), si la transparence permet d'accroître la stabilité du système financier pendant les crises, elle a toutefois des effets potentiellement déstabilisants en période normale. Cependant, dans la mesure où la transparence signale une détérioration des fondamentaux, il existe un problème d'engagement (commitment) pour le régulateur : tandis qu'une politique de diffusion d'information est optimale ex ante, le régulateur aura une incitation ex post à ne pas révéler d'information.

Goldstein et Leitner [2013] étudient également la politique optimale de diffusion de l'information d'un régulateur qui a de l'information à propos de la capacité des banques à faire face à des chocs de liquidité mais en se concentrant sur l'arbitrage suivant : fournir de l'information est nécessaire pour prévenir l'effondrement du marché, mais diffuser trop d'information détruit les opportunités de partage du risque. Ils montrent ainsi qu'en temps normal, ne pas diffuser l'information est optimal, tandis qu'en temps de crise une diffusion partielle est optimale.

Spargoli [2012] prétend que le niveau socialement optimal de diffusion d'information par les superviseurs dépend de leur capacité à contraindre les 'mauvaises' banques à se recapitaliser. Il étudie l'arbitrage auquel est confronté le régulateur entre diffuser ou non les pertes en capital des banques en période de crise. L'annonce contraint les banques à réduire leur risque de défaut, mais les conduit à réduire leur activité, à moins que le régulateur ne les recapitalise. Dans un tel contexte, un régulateur qui ne peut pas recapitaliser les banques préfèrera divulguer moins d'information si les coûts de la réduction de l'activité (baisse d'effectifs) sont plus importants que 
les coûts de défaut attendus. Dans le cas contraire, ou si le régulateur est en mesure de recapitaliser les banques, la baisse des fonds propres des banques sera pleinement révélée.

Selon Schuermann [2012], le type d'information diffusée au moyen des tests de stress devrait dépendre de l'état de l'industrie bancaire. En effet, si les investisseurs paniquent et ne croient pas dans les bilans bancaires parce qu'ils sont extrêmement opaques, les tests de stress devraient clarifier leur situation en donnant des détails pour chaque banque, permettant ainsi de restaurer la confiance. Une fois la confiance restaurée et étant donné le coût de diffuser des tests donnant de l'information spécifique pour chaque banque, une information agrégée pourrait suffire.

\section{Quelles leçons peut-on tirer des études empiriques?}

Les tests de stress et leur impact sur le marché ont été étudiés empiriquement.

Peristiani et al. [2010] étudient le cas du test de stress effectué par les federal bank supervisors en 2009 et montrent que ce test a permis de limiter la panique financière en produisant de l'information notamment sur l'ampleur des difficultés financières des banques.

Cardinali et Nordmark [2011] étudient la réaction du marché action aux tests de stress européens de 2010 et 2011, en considérant les revenus anormaux cumulés pour les banques testées et non testées. Ils montrent d'une part que les résultats de 2010 étaient relativement peu informatifs et d'autre part que l'annonce de la méthodologie utilisée en 2011 a déclenché des revenus anormaux cumulés négatifs pour les banques soumises à des tests de stress tandis que celles qui n’y avaient pas été soumises ont été relativement peu affectées. Enfin, ils ne trouvent pas de différence entre les banques faisant partie des PIIGS $^{9}$ et les autres.

Ellahie [2012] considère également les tests de stress européens de 2011 pour évaluer dans quelle mesure l'annonce des résultats a conduit à un changement dans les asymétries d'information. Il trouve que les annonces des stress tests n'ont pas d'incidence significative sur les mesures de l'asymétrie d'information ou de l'incertitude d'information pour les banques testées. Il trouve aussi que, lors de la divulgation des résultats des tests de 2011, l'asymétrie d'information baisse pour les banques testées alors que l'incertitude informationnelle augmente indiquant soit une imprécision des informations révélées, soit une aggravation de la crise de la dette souveraine. En outre, il montre que l'annonce d'exposition en cas de défaut dans les résultats des tests de 2011 contenait de l'information pour mesurer les asymétries d'information, l'incertitude informationnelle, les spreads et les prix des actions. Des tests de stress transparents permettraient ainsi d'améliorer la qualité de l'information sur les marchés financiers pendant les crises.

Bischof et Daske [2012] montrent que la diffusion des résultats des tests de stress en Europe a réduit l'opacité des banques (i.e. a augmenté la diffusion volontaire d'information par les banques) ainsi que la prise de risque des banques lorsque ces tests montraient des résultats négatifs.

Enfin, Petrella et Resti [2013] considèrent les évolutions de prix rencontrées par les banques européennes après la diffusion des résultats des stress tests en 2011 et montrent que ces tests ont 
produit une information utilisable par les participants au marché et ont pu réduire l'opacité des banques.

\section{Politique monétaire non-conventionnelle, communication et transparence}

Depuis le milieu des années 90, les banques centrales ont placé au cœur de leur stratégie la question de la transparence en matière de diffusion d'information. Comme l'a suggéré l'expérience d'Alan Greenspan - gouverneur de la banque centrale américaine entre août 1987 et janvier 2006 - en matière de communication avec les marchés, la recherche d'une transparence accrue est perçue comme un moyen d'améliorer l'efficacité de la politique monétaire (Woodford, [2005]). Il s'agit notamment d'influencer les anticipations des agents privés concernant les taux d'intérêt futurs (Blinder, [1998] ; Fracasso et al., [2003]).

Depuis fin 2008, les banques centrales, avec à leur tête la Fed, ont été amenées à recourir à ce qu'il est convenu d'appeler des outils de politique monétaire non-conventionnelle, et ce, en lien avec le fait que le taux d'intérêt directeur s'est retrouvé à un niveau proche de 0 . Plus précisément, avec la crise financière et ses répercussions sur l'économie réelle, les banques centrales sont confrontées à un risque de déflation ou à une situation proche de la trappe à liquidité, elles peuvent encore agir dans trois directions identifiées par Bernanke [2009]. En premier lieu, elles peuvent chercher à ancrer et modeler les anticipations de taux d'intérêt. En effet, les taux d'intérêt à court terme et à long terme intègrent les anticipations concernant les évolutions des taux directeurs. Lorsque ces derniers sont proches de 0 , la seule incertitude porte sur le moment où la banque centrale va les accroître, ce qui peut empêcher toute baisse des autres taux. En conséquence, pour stimuler l'économie, la banque centrale peut s'engager explicitement à laisser ses taux directeurs à 0 pour une certaine durée. Les anticipations étant ainsi ancrées, il doit en résulter une baisse des taux longs. En deuxième lieu, les banques centrales peuvent modifier la composition des actifs qu'elles détiennent dans leur bilan. Elles peuvent agir soit en modifiant la maturité (dans le sens d'un allongement afin d'agir sur le niveau des taux d'intérêt de maturité plus longue), soit en diversifiant les actifs achetés au-delà des titres émis par les Etats (elles soutiennent ainsi les prix et ont une action sur la liquidité des marchés). Enfin, les banques centrales peuvent augmenter la taille de leur bilan par le biais d'une politique dite d'assouplissement quantitatif (quantitative easing). Les banques centrales ont alors un objectif de croissance de la base monétaire. Elles achètent massivement des titres lors des opérations de refinancement, d'où l'accroissement de la taille des bilans. Ces trois axes d'interventions sont au cœur des politiques monétaires non-conventionnelles (Pattipeilohy et al, [2013]).

L'utilisation d'instruments non traditionnels de la politique monétaire appelle une communication plus importante sur les intentions de la banque centrale. L'axe central est alors de fournir des informations sur le sentier le plus vraisemblable des taux d'intérêt à court terme concernant le futur afin d'ancrer les anticipations pour empêcher des tensions sur les taux longs (Yellen, [2011] et [2013]). Cette stratégie prend appui sur deux piliers qu'il convient de distinguer. Le premier fait 
référence à la communication prospective ou communication avancée (forward guidance) relative aux taux d'intérêt. Le second fait référence à la communication par la banque centrale à propos de sa politique d'achats massifs de titres sur certains segments des marchés financiers. L'efficacité d'une telle politique repose sur la diffusion d'informations concernant, d'une part, le programme d'achats de titres et, d'autre part, la trajectoire de long terme la plus vraisemblable concernant la détention des titres ainsi acquis.

\section{La politique de forward guidance}

Cette politique s'inscrit dans une stratégie de communication qui vise à influencer durablement les anticipations sur l'évolution des prix à moyen et long terme. ${ }^{10}$ Cet outil consiste à guider les anticipations des investisseurs sur l'évolution des prix à moyen et long terme, à l'aide d'une communication active. L'objectif est d'améliorer la transparence de la politique monétaire afin d'encourager l'investissement et la consommation. Plus précisément, la politique de forward guidance repose sur l'engagement explicite d'un niveau de taux d'intérêt maximal, indépendamment de la marge d'erreur anticipée dans les prévisions économiques : elle consiste à annoncer et à s'engager sur la trajectoire future du taux directeur. Il s'agit d'un engagement sur la conduite des taux d'intérêt, alors que la banque centrale ne se «pré-engage » habituellement pas, le niveau des taux devant pouvoir être ajusté à tout moment en cas de choc sur l'économie de nature à l'affecter à un horizon pertinent pour la conduite de la politique monétaire. Cette politique explicite la stratégie de la banque centrale, mais surtout renforce sa crédibilité, donc l'efficacité des canaux de transmission de la politique monétaire. Par cet intermédiaire, les banques centrales souhaitent accroître la transparence de leur action et ancrer les anticipations.

La mise en œuvre de la politique de forward guidance

Aux Etats-Unis, la mise en œuvre de la politique de forward guidance a été initiée dès décembre 2008. Ainsi, la réunion du Federal Open Market Committee (FOMC) a communiqué aux marchés les conditions sous lesquelles les taux directeurs resteraient dans la fourchette $0-0.25 \%$ en précisant que «les conditions économiques sont susceptibles de justifier des niveaux exceptionnellement bas du taux des fonds fédéraux pour un certain temps $\gg .{ }^{11}$ En mars 2009, la communication du FOMC commence à évoluer : le terme «for some time » a été changé par «for an extended period ». Le FOMC souligne aussi que le sentier futur des Fed Funds est dépendant de l'évolution future de l'activité et fournit aux marchés des informations sur les éléments pouvant conduire dans le futur à un changement de politique monétaire. Par exemple, dans son communiqué de novembre 2009, le Comité de politique monétaire précise que les conditions économiques guidant les orientations de la politique monétaire portent sur un faible taux d'utilisation des capacités de production, une inflation tendancielle modérée et des anticipations d'inflation stables (Yellen, [2011]). Cependant, la communication du FOMC demeure conditionnée à une période de temps comme le suggère le communiqué d'août 2011 où il est fait explicitement référence à une date pour le maintien des taux d'intérêt directeurs (mi-2013). Comme le rappellent English et al. 
[2013], le FOMC a redouté que cette approche du forward guidance soit mal comprise par les agents économiques. C'est la raison pour laquelle la communication du FOMC a connu un changement important à partir de décembre 2012. Plus précisément, le FOMC a décidé de conditionner son engagement sur l'évolution future des taux à des seuils conjoncturels déclencheurs. Ainsi, il s'est engagé à ce que les taux restent bas tant que le taux de chômage demeurera au-dessus de 6,5\% et que l'inflation prévue à 1-2 ans ne dépassera pas de plus d'un demi-point l'objectif à long terme de $2 \%$ et que les anticipations d'inflation à plus long terme resteront bien ancrées. ${ }^{12}$ Il est important de souligner que cette évolution dans la communication du FOMC s'inscrit dans un processus plus large d'effort de clarification de la politique monétaire. Ainsi, depuis novembre 2007, le FOMC publie un Summary of Economic Projections (SEP) fournissant des informations détaillées sur les prévisions de ses membres. ${ }^{13}$ Le SEP permet aux agents économiques de capter des informations sur les objectifs à long terme du FOMC concernant le chômage et l'inflation ainsi que sur la stratégie suivie. En janvier 2012, le FOMC a publié une déclaration concernant les objectifs à long terme et la stratégie de politique monétaire (Statement on Longer-Run Goals and Monetary Policy Strategy). Pour la première fois, le FOMC a communiqué sur une valeur numérique précise concernant l'objectif d'inflation à long terme $(2 \%) .{ }^{14}$ En outre, la crise financière a conduit le FOMC à rallonger et rendre plus complexe ses déclarations d'après réunions. Ainsi, alors qu'en 2006 les déclarations comptaient en moyenne 200 mots, elles comportent près de 600 mots en 2013 (English et al. [2013]). Les déclarations du Comité contiennent une discussion plus approfondie sur l'orientation de la politique et des conditions sous-jacentes guidant la politique monétaire future.

La BCE s'est engagée plus tardivement dans la stratégie du forward guidance. En effet, il faut attendre la conférence de presse donnée par Mario Draghi, gouverneur actuel de la BCE, suivant la réunion mensuelle du Conseil des Gouverneurs du 4 juillet 2013 pour voir émerger une communication qui peut s'apparenter à cette stratégie. Ainsi, il est notamment affirmé que «le Conseil des gouverneurs s'attend à ce que les taux d'intérêt directeurs restent à leur niveau actuel ou plus bas pour une période prolongée $\gg .{ }^{15}$ Cette nouvelle communication de la BCE est en partie liée à la réduction de ses marges de manœuvre à la suite de sa décision de baisser son taux directeur à $0,50 \%$ en mai 2013 puis $0,25 \%$ en novembre 2013. Elle répond aussi à l'augmentation de la volatilité des taux courts au début de l'été 2013 et à la volonté de la BCE d'ancrer plus fermement les anticipations de taux des agents privés (Coeuré, [2013]). Cependant, cette stratégie de forward guidance a une portée encore limitée. D'une part, la BCE entretient une certaine ambiguïté en conditionnant son engagement à une période de temps sans référence à des variables économiques seuils. D'autre part, comme le rappelle Coeuré [2013], le forward guidance adopté reste ancré au mandat de la banque centrale et à son cadre opérationnel. 


\section{Quelle efficacité ?}

L'efficacité de la politique de forward guidance fait l'objet de débats importants, tant sur les plans théorique qu'empirique, débats qui remontent à l'expérience japonaise des années $90 .{ }^{16}$

Krugman [1998] considère que lorsque le taux directeur atteint le seuil limite de $0 \%$ en terme nominal, la banque centrale doit s'engager dans un «principe d'irresponsabilité » (irresponsibility principle). Selon ce principe, la banque centrale s'engage à laisser les prix augmenter afin d'accroître les anticipations d'inflation des agents privés. Les modèles issus de la nouvelle économie keynésienne adoptent un point de vue très proche. Ainsi, Eggertsson et Woodford [2003] recommandent que la banque centrale s'engage, de préférence de manière explicite, à maintenir ses taux directeurs à un niveau bas ou nul pendant une durée suffisante pour que certaines conditions prédéterminées relatives à la croissance et à l'inflation soient réunies. Si l'engagement est jugé crédible, deux effets expansionnistes sur l'activité sont attendus. En premier lieu, si les agents privés anticipent une inflation future plus élevée, alors ils sont incités à accroître leurs dépenses dès aujourd'hui, ce qui doit induire une hausse immédiate des prix. Le point important est que cet effet est amplifié si les agents considèrent que la banque centrale laissera inchangé son taux d'intérêt même si l'inflation augmente. Comme le souligne Woodford [2013], plus la banque centrale s'engage à maintenir constant son taux directeur pendant une longue période, plus l'effet d'amplification est important. En second lieu, en application de la théorie de la structure par terme des taux d'intérêt, l'anticipation de taux courts futurs bas conduit à des taux d'intérêt longs futurs plus faibles, ce qui doit favoriser la demande de crédit et, par là-même, la consommation et l'investissement. Walsh [2008] considère que lorsque la banque centrale détient une information supérieure sur les conditions économiques que celle détenue par les agents privés, la politique de forward guidance améliore le bien-être même si la politique monétaire est discrétionnaire. On peut ici rappeler le travail de Romer et Romer [2000] qui a montré que la banque centrale américaine avait eu de meilleurs résultats en termes de prévisions que ceux obtenus par des institutions privées. Selon les auteurs, ce résultat s'explique par le fait que la banque centrale a accès à des informations privilégiées.

Ainsi, le débat théorique suggère que la politique de forward guidance peut être efficace pour accroître l'activité économique si cette politique est jugée crédible par les agents et si la banque centrale détient une information plus précise que les agents privés. Ces deux caractéristiques font débat. Ainsi, Clarida [2012] a souligné que si les agents privés confondent l'engagement des autorités avec une politique discrétionnaire, alors le forward guidance n'a pas d'effet sur les taux longs. En effet, dans ce cas, les agents privés anticipent que les taux courts sont bas (élevés) parce que l'activité économique est faible (élevée). De son côté, Fratzscher [2013] considère que cette politique présente un certain nombre de risques. Plus précisément, les annonces des banques centrales ayant pris une telle importance pour les marchés financiers, les variations de prix traduiraient davantage la réaction des marchés financiers aux déclarations des banques centrales, 
qu'aux réalités financières et économiques. ${ }^{17}$ Il en résulte que les sources d'information privées sont occultées, privant l'autorité monétaire d'un point de vue indépendant de la part des acteurs de marché, utile à la conduite de sa politique. Enfin, lorsque les banques centrales orientent les marchés à l'excès dans une certaine direction, elles risquent de perdre leur crédibilité. L'argument sur les interactions entre informations privées et informations diffusées par la banque centrale fait une nouvelle fois écho aux travaux en termes de jeux de coordination à la Morris et Shin [2002] et introduits plus haut. Comme nous l'avons déjà évoqué, cette littérature s'interroge sur le bien-fondé de la transparence ${ }^{18}$. Dans cette perspective, la politique de forward guidance pourrait être déstabilisante.

Les travaux empiriques sur l'efficacité de la politique de forward guidance conduisent à des conclusions assez mitigées. Yellen [2011] compare la valeur moyenne trimestrielle des anticipations des fonds fédéraux à des horizons de 0 à 6 trimestres à deux dates différentes : le $1^{\text {er }}$ décembre 2008 et le $1^{\text {er }}$ février 2009. Les données sont issues du consensus des prévisionnistes professionnels publié dans les Blue Chip Surveys. Elle interprète la baisse attendue plus importante des taux courts entre les deux dates comme le signe de l'efficacité de la politique de forward guidance. English et al. [2013] simulent un modèle de l'économie américaine afin de tester différentes alternatives en termes de forward guidance. Un résultat particulièrement important est que cette politique est d'autant plus efficace qu'elle est conditionnée à des valeurs seuils d'indicateurs économiques.

Kool et Thornton [2012] évaluent l'efficacité de la politique de forward guidance aux Etats-Unis, en Nouvelle-Zélande, en Norvège et en Suède. Ils cherchent à répondre à deux questions. En premier lieu, la politique de forward guidance permet-elle d'améliorer la prédictibilité des taux courts? En second lieu, en prenant appui sur la théorie de la structure par terme des taux d'intérêt selon laquelle une meilleure prédictibilité des taux courts doit se transmettre à l'ensemble de la courbe des taux, la politique de forward guidance augmente-t-elle la capacité des agents à prévoir les taux longs? Les résultats qu'ils obtiennent conduisent à nuancer sérieusement les travaux qui considèrent que les banques centrales peuvent mener des politiques monétaires efficaces en termes de forward guidance. En effet, si cette politique permet d'améliorer la capacité des agents privés à prévoir les taux courts futurs pour des horizons de prévisions inférieurs à un an, elle n'a aucune influence sur la prédictibilité des taux à plus long terme. Or, c'est ce segment de la courbe des taux qui influence les principales décisions économiques des agents.

\section{La politique d'achats massifs de titres par les banques centrales}

La politique d'achats massifs de titres par les banques centrales représente un pilier important des politiques monétaires non-conventionnelles mises en œuvre à la suite de la crise financière. Cette politique prend appui sur le fait que les prix et les rendements des actifs sont influencés par les achats courants de titres. Cependant, il convient de relever que les anticipations concernant les achats futurs exercent aussi une influence sur ces prix et ces rendements. C'est dans cette 
perspective que la politique de communication de la banque centrale trouve toute son importance. En effet, à travers sa politique de communication sur ses achats présents et futurs, la banque centrale peut exercer une influence sur les conditions financières prévalant sur les marchés financiers.

Une Banque Centrale Européenne en retrait

Si toutes les banques centrales des principaux pays développés se sont engagés dans des programmes d'achats de titres afin, d'une part, de restaurer les conditions d'un fonctionnement normal de certains segments des marchés financiers, et, d'autre part, de rendre plus efficace la transmission de la politique monétaire ${ }^{19}$, il convient de souligner que les montants en jeu diffèrent. La Réserve Fédérale américaine a mis en œuvre trois programmes d'achats de titres (Bernanke [2012], English et al. [2013] et Woodford [2013]). Ces programmes correspondent aux opérations de quantitative easing qui se sont succédées depuis le début de la crise financière. Le premier programme a été annoncé en novembre 2008. La banque centrale a déclaré être prête à acheter jusqu'à 1175 milliards de dollars de titres (dont 1125 milliards de titre adossé à des créances hypothécaires (mortgage-backed security, MBS)) jusqu'en mars 2010. Le deuxième programme d'achats a été lancé en novembre 2010. La banque centrale américaine a annoncé son intention d'acquérir jusqu'à 600 milliards de titres longs d'ici juin 2011. Cette annonce a été complétée en septembre 2011 par un programme d'extension de la maturité des titres détenus (Maturity extension program (MEP)) dans lequel la banque centrale s'engage dans l'achat de titres longs du Trésor en contrepartie de ventes équivalentes de titres courts. A fin 2012, plus de 650 milliards de dollars de titres longs ont ainsi été achetés. Le dernier programme a été lancé en septembre 2012 avec l'annonce d'achats de titres MBS d'agences sponsorisées par le gouvernement ${ }^{20}$ pour un montant de 40 milliards par mois. La spécificité de ce programme est de ne contenir aucune date précise d'arrêt puisqu'il est conditionné à une amélioration significative du marché du travail.

En octobre 2010, la Banque du Japon a lancé un programme d'achats de titres privés et publics d'un montant de 101000 milliards de yen d'ici à fin 2012, soit près de $20 \%$ du PIB. Il a été remplacé en avril 2013 par un nouveau programme d'assouplissement quantitatif (Quantitative and Qualitative Easing Program) ayant pour objectif de doubler la taille du bilan de la banque centrale d'ici fin 2014. Ce programme a notamment pour objectif d'allonger la maturité des titres du gouvernement détenus par la banque centrale (English et al. [2013]). La Banque d'Angleterre a lancé deux programmes successifs d'achats de titres (mars 2009 - juin 2010 et octobre 2011 octobre 2012) pour un montant de 375 milliards de livres sterlings (près de $25 \%$ du PIB). Ces achats ont porté quasi-exclusivement (99\%) sur des obligations de l'Etat britannique (gilts). La BCE est restée largement à l'écart de ces politiques d'achats massifs. Cependant, trois principaux programmes peuvent être identifiés. Le premier porte sur le programme d'achat d'obligations sécurisées (Covered Bonds Purchase Program) qui a conduit la BCE à acquérir pour 76 milliards d'euros de titres entre mi-2009 et fin 2012. En mars 2010, la banque centrale a lancé un programme 
pour les marchés de titres (Securities Market Programme, SMP). Il convient de noter que ce programme ne contient aucun objectif explicite en termes de volumes d'actifs achetés ou de rendements à atteindre. Entre la date de lancement et août 2012, elle a acquis près de 208 milliards d'euros de titres. Ce programme a alors été remplacé par les opérations monétaires sur titres (Outright Monetary Transactions, OMT) sur les marchés secondaires des obligations souveraines.

$\underline{\text { Par quels canaux de transmission les politiques d'achats de titres influencent-elles les }}$ taux d'intérêt?

Les politiques d'achats massifs par les banques centrales peuvent exercer une influence sur les rendements des titres par quatre canaux principaux.

Le premier est le canal du portefeuille (portfolio balance channel) selon lequel les achats de titres peuvent exercer un impact sur la composition des portefeuilles du secteur privé (Gagnon et al. [2011] et Pattipeilohy et al. [2013]). Plus précisément, en achetant de manière massive certains titres, la banque centrale tend à réduire les montants détenus par les agents privés. Dans le même temps, lors de ces opérations d'achats, la banque centrale augmente le montant des actifs liquides à la disposition des agents privés. Afin que ces derniers acceptent d'ajuster leurs portefeuilles, il faut que les rendements des titres acquis par la banque centrale diminuent. Autrement dit, ces opérations doivent induire une hausse de leur prix, soit aussi une baisse de leur taux d'intérêt.

Le deuxième canal est l'effet de liquidité qui prend deux formes. D'une part, dans la mesure où les achats de la part de la banque centrale créent une nouvelle source de demande, cela incite d'autres investisseurs à venir sur les marchés (Gagnon et al. [2011]). D'autre part, en injectant de la liquidité en contrepartie des achats de titres, la banque centrale exerce une influence sur le niveau des taux d'intérêt à très court terme sur le marché interbancaire (Pattipeilohy et al. [2013]).

Les deux derniers canaux - identifiés par Krishnamurthy et Vissing-Jorgensen [2011] - sont particulièrement importants pour notre propos en raison de leurs liens avec la politique de forward guidance.

Le troisième canal repose en effet sur un effet signal (signalling effect) selon lequel les achats de titres par la banque centrale s'accompagnent de l'anticipation par les agents privés d'une baisse des taux longs. Ce canal repose fondamentalement sur la crédibilité de l'engagement de la banque centrale à ne pas modifier sa politique monétaire dans le futur, notamment si des tensions sur l'inflation apparaissent. Bernanke et al. [2004] soulignent que la politique d'achats massifs de titres par la banque centrale renforce la crédibilité de l'engagement à maintenir à un faible niveau les taux courts. Le raisonnement est le suivant: si les autorités monétaires devaient augmenter leur taux d'intérêt, alors elles subiraient les conséquences négatives en termes de pertes en capital en raison de la hausse des taux longs qui résulteraient de l'anticipation de celle des taux courts. Cecioni et al. [2011] nuancent ce point de vue en soulignant, d'une part, que les pertes potentielles de la banque centrale ne porteraient que sur les titres non détenus jusqu'à leur l'échéance et, d'autre part, que la banque centrale n'est pas une institution privée ayant comme objectif principal la 
rentabilité. Dans ce contexte, l'effet signal repose de manière déterminante sur le policy guidance de la politique monétaire.

\section{L'efficacité des politiques d'achats massifs de titres}

Gagnon et al. [2011] utilisent une approche en termes d'événements (event studies) pour analyser la réponse des taux d'intérêt à huit annonces de la Fed entre novembre 2008 et novembre 2009. Les annonces considérées contiennent des informations sur la taille (effective ou potentielle) du programme, sa composition et / ou sa durée. Plus précisément, ils étudient la réaction des taux d'intérêt suivants sur la période décembre 2008 - décembre 2010 : les rendements des titres du Trésor à deux et dix ans, les rendements des titres de la dette des agences sponsorisées à dix ans, le rendement des coupons courants des titres MBS des agences à trente ans, la prime à terme à dix ans des titres du Trésor, le swap de taux à dix ans et les rendements des obligations des entreprises non financières notées Baa. Les deux dernières variables permettent de voir dans quelle mesure d'autres taux que ceux liés à des actifs acquis par la banque centrale sont affectés par la politique d'achats massifs d'actifs. La réponse des taux d'intérêt est estimée en utilisant une fenêtre d'un jour autour des annonces. Ainsi, les auteurs étudient la variation des taux d'intérêt entre le niveau atteint à la fermeture des marchés la veille de l'annonce et leur niveau à la fermeture des marchés le jour de l'annonce. Le résultat principal de Gagnon et al. [2011] est que, conformément aux attentes, les annonces sont suivies d'une baisse des taux d'intérêt.

Krishnamurthy et Vissing-Jorgensen [2011] étudient les réponses des taux d'intérêt les jours des annonces des programmes en distinguant entre les segments des marchés. Par rapport à l'analyse de Gagnon et al. [2011], ils utilisent des données intra-journalières afin d'identifier les effets sur les prix et les volumes échangés dans les minutes qui suivent les annonces. Davantage de canaux de transmission sont envisagés, plus particulièrement un effet signal et l'impact des annonces sur les taux d'inflation anticipés et sur le degré d'incertitude concernant l'inflation future. Leurs principaux résultats sont les suivants. En premier lieu, ils montrent que le Quantitative Easing 1 (QE1) a conduit à une révision à la baisse concernant les taux des fonds fédéraux futurs les jours des annonces. Autrement dit, les annonces sont suivies d'un glissement vers le bas de la courbe des taux implicites. Le Quantitative Easing 2 (QE2) suggère des résultats similaires même si les effets sont moins importants. Ainsi, un premier résultat important est que l'effet signal est particulièrement important. En second lieu, Krishnamurthy et Vissing-Jorgensen [2011] montrent, en observant le comportement des titres indexés sur l'inflation à la suite des annonces, que les anticipations d'inflation augmentent - ce qui conduit à une baisse des taux réels anticipés - et que l'incertitude sur l'inflation future se réduit. Ce dernier résultat suggère que le canal de l'inflation est significatif. Surtout, la réduction de l'incertitude sur l'inflation future signifie que les agents privés anticipent moins de pressions déflationnistes à la suite des annonces des programmes d'achats de titres. 
En ce qui concerne la zone euro, Falagiarda et Reitz [2013] trouvent que la communication de la BCE concernant ses mesures de politique monétaire non-conventionnelle a contribué au recul du spread souverain de l'Italie vis-à-vis de l'Allemagne entre 2008 et 2012. Les auteurs montrent que l'effet a été plus important sur la période 2010-2012 relativement à la période 2008-2009 suggérant une efficacité importante du SMP puis de l'OMT.

Pattipeilohy et al. [2013] utilisent des données de panel incluant les rendements journaliers des titres, des spreads sur les CDS et d'autres variables financières (indices boursiers et indicateurs de volatilité) sur la période juin 2010 - juin 2012. Ils étudient l'impact des différentes politiques mises en œuvre par la $\mathrm{BCE}^{21}$ et les interventions du Fonds européen de stabilité financière en focalisant leur attention sur l'Espagne, l'Italie, l'Irlande, la Grèce et le Portugal. Les résultats obtenus montrent que les opérations de refinancement à long terme ont eu un impact favorable sur les rendements des obligations souveraines. A contrario, les effets du programme pour les marchés de titres (SMP) ne sont que de très court terme.

\section{4 - Conclusion}

Cet article a proposé une analyse de la crise financière internationale en considérant la question de l'information, vue à la fois du côté des marchés et de la réponse des autorités à cette crise. Il montre l'importance de produire de l'information pour que les marchés fonctionnent correctement dans un contexte où les participants au marché (i) peuvent parfois ignorer les informations pertinentes, (ii) n'ont pas forcément d'incitation à produire de l'information et (iii) peuvent surréagir aux annonces. Dans la mesure où ces situations peuvent apparaître successivement et générer des phases de boom-bust, il est important pour les autorités de tenir compte du contexte et du type d'information à produire. Ainsi, la réponse des autorités doit-elle être adaptée au contexte et à la réaction potentielle des autres participants au marché associée à ce contexte dans un jeu stratégique et dynamique.

Les tests de stress représentent un bon outil pour limiter la myopie au désastre et répondre au déficit en matière de production d'information. La question de leur diffusion optimale est liée à la façon dont le marché les interprète et réagit à une situation de connaissance commune de l'état de l'industrie bancaire et financière. Plus précisément, si les autorités peuvent internaliser les externalités négatives dues aux déficiences des marchés en matière de production d'information, elles doivent tenir compte du fait que l'information publique peut avoir un effet déstabilisant. En effet, comme souligné dans l'approche en termes de concours de beauté, lorsque l'on considère la transparence, il ne faut pas oublier que publier des signaux préalables concernant les problèmes potentiels dans certaines parties du système financier peuvent générer des paniques et conduire à une crise financière (Gai et Shin [2003]). Dans une situation où la crise financière se propage à l'économie réelle, les outils de politique monétaire non-conventionnelle en lien avec la politique de communication ont un rôle à jouer en matière de gestion des anticipations. La politique de 
communication optimale doit tenir compte de la réaction du marché. Il est ainsi essentiel que la banque centrale communique avec les marchés sur la fin programmée de ces politiques nonconventionnelles, et ce, afin d'éviter une sur-réaction des agents privés pouvant conduire à un accroissement de l'instabilité et / ou à une augmentation brutale des taux d'intérêt.

$\mathrm{Au}$ total, confrontées aux inefficiences informationnelles présentes sur les marchés, cet article souligne que les autorités doivent faire face à un double écueil : produire et diffuser de l'information afin de guider les marchés sans pour autant engendrer des paniques aux conséquences déstabilisatrices. L'expérience récente des autorités de régulation et des banques centrales suggère que la politique de communication est susceptible d'éviter l'écueil des conséquences déstabilisatrices d'une plus grande transparence. Deux exemples illustrent ce point de vue. D'une part, comme l'ont souligné, entre autres, Cardinali et Nordmark [2011], le contenu informationnel des tests de stress des banques exerce une influence sur la capacité des autorités à influencer les marchés. Rappelons que ces auteurs ont montré que les tests réalisés dans l'Union européenne en 2011, au contenu informationnel plus précis, ont davantage impacté les marchés que ceux réalisés en 2010. D'autre part, l'introduction de valeur seuil pour les variables macroéconomiques clefs permet aux banques centrales de guider les anticipations des agents privés à la fois sur la poursuite des politiques monétaires non-conventionnelles mais aussi, et surtout, sur leur date probable de sortie. 
* Jean-Pierre Allegret est Professeur à l'Université Paris Ouest Nanterre la Défense et rattaché au laboratoire ECONOMIX. ECONOMIX - UMR 7235 CNRS et Université Paris Ouest Nanterre la Défense, 200 Avenue de la République, 92001 Nanterre cedex France; e-mail: jallegret@u-paris10.fr.

** Camille Cornand est chargée de recherche au CNRS rattachée au laboratoire GATE - UMR 5824 CNRS. Université de Lyon, Lyon, F-69007, France ; CNRS, GATE Lyon Saint-Etienne, Ecully, F-69130, France ; email: cornand@gate.cnrs.fr.

${ }^{1}$ Les subprimes sont des crédits hypothécaires à des ménages très risqués.

${ }^{2}$ Rappelons qu'un fait est de connaissance commune lorsque chacun le sait, lorsque chacun sait que chacun le sait, et ainsi de suite.

${ }^{3}$ Il est dorénavant bien établi en psychologie cognitive que les agents économiques ont tendance à fonder leur règle de décision sur des heuristiques simples ou des règles arbitraires, voir en ce sens Kahneman et al. [1982] pour des études expérimentales.

${ }^{4}$ Voir Section 3 pour davantage de détails.

${ }^{5}$ Le concours de beauté a été introduit par Keynes [1936]. Les participants au marché tentent de deviner la valeur des fondamentaux : ils cherchent à choisir des actions appropriées étant donné l'état de l'économie. Mais ils s'engagent également dans un jeu à somme nulle pour deviner les actions des autres agents, le gain d'un joueur dépendant de la distance entre son action et l'action des autres. Plus la distance est petite, plus le gain est grand. Il existe donc un motif de coordination pour les preneurs de décision et un motif fondamental. Ainsi, l'action d'un investisseur dépend-elle non seulement du fondamental de l'économie, mais aussi de ce que les autres décident de faire.

${ }^{6}$ Plus précisément, le modèle est testé sur trois sous-périodes : (i) avant le début de la crise en juillet 2007 ; (ii) d'août 2007 à la faillite de Bear Stearns en mars 2008 et (iii) après la faillite de cette banque jusqu'à l'effondrement de Lehman Brothers en septembre 2008.

7 Par exemple, le Dodd-Frank Wall Street Reform and Consumer Protection Act requiert du Board of Governors de la Réserve Fédérale américaine qu'il fasse subir des tests de stress annuels aux institutions financières systémiquement importantes.

${ }^{8}$ Le Supervisory Capital Assessment Program (SCAP) mis en œuvre en 2009 en est un bon exemple. En 2009, les Etats-Unis ont connu une très grande incertitude sur la santé de leur système bancaire. En l'absence d'une compréhension concrète et crédible des problèmes relatifs aux bilans bancaires, les investisseurs étaient réticents à prêter. Seule une évaluation crédible des pertes face à un scénario de stress macroéconomique par les superviseurs ont permis de restaurer la confiance. En Europe, le Committee of European Bank Supervisors (CEBS) a conduit un premier test de stress sur 91 banques européennes en 2010 puis un second sur 90 banques en 2011.

${ }^{9}$ Espagne, Irlande, Italie, Grèce et Portugal.

${ }^{10}$ Comme le rappellent Svensson [2010] et Antonin et al. [2013], avant même la crise financière de 2008, certaines banques centrales (Nouvelle-Zélande depuis 1997, Norvège depuis 2005 et Suède depuis 2007) avaient déjà mis en œuvre une telle stratégie. Les Etats-Unis avaient également mis en place cette stratégie de communication à plusieurs reprises lorsque les taux étaient très bas et le Federal Open Market Committee (FOMC) avait introduit de manière implicite la politique de forward guidance dès août 2003.

11 «Economic conditions are likely to warrant exceptionally low levels of the federal funds rate for some time».

12 La Banque d'Angleterre conditionne elle aussi, depuis août 2013, sa politique de taux d'intérêt bas à l'évolution du taux de chômage (en spécifiant un seuil de 7 \%) en précisant qu'une inflation contenue autour de $2 \%$ demeure déterminante dans la conduite de la politique monétaire. Voir Antonin et al. [2013].

${ }^{13}$ Les quatre variables clefs intégrées dans le SEP reflètent le mandat dual de la Réserve Fédérale : le taux de croissance du PIB réel, le taux de chômage, le taux d'inflation et le taux d'inflation sous-jacente. Pour plus de développements, voir English et al. [2013].

${ }^{14}$ Aucune cible précise n'est donnée en ce qui concerne un objectif de taux de chômage à long terme.

15 «The Governing Council expects the key interest rates to remain at present or lower levels for an extended period of time ».

${ }^{16}$ Voir aussi Cecioni et al. [2011].

17 Fratzcher [2013] relève ainsi : «lorsque les responsables politiques promettent d'agir face à certains risques, invariablement les marchés minorent leurs conséquences. En mai dernier, Bernanke a lancé un avertissement sérieux concernant les prises de risque excessives sur les marchés financiers. Cela n'a pas empêché les investisseurs de pousser les indices boursiers à des sommets en dépit d'un redémarrage faible et 
incertain, tandis que l'indice de volatilité VIX qui traduit la perception des risques par les investisseurs chutait à un niveau plancher jamais atteint depuis le boom des années 2005 et 2006 ».

${ }^{18}$ Pour une revue de la littérature, voir Allegret et Cornand [2006].

${ }^{19}$ Ainsi, la BCE précise que le programme d'opérations monétaires sur titres annoncé en août 2012 a «pour double objectif de sauvegarder un mécanisme de transmission approprié de la politique monétaire et de préserver l'unicité de la politique monétaire à travers la zone euro en apportant un soutien pleinement efficace permettant d'éviter tout scénario néfaste pouvant gravement compromettre la stabilité des prix dans la zone euro ».

${ }^{20}$ Notamment Freddie Mac et Fannie Mae.

${ }^{21}$ Pattipeilohy et al. [2013] prennent ainsi en compte (i) les injections de liquidité par l'intermédiaire des opérations principales de refinancement, (ii) le programme pour les marchés de titres (Securities Market Programme) et (iii) les opérations de refinancement à long terme (long-term refinancing operations, LTROs) mises en œuvre en décembre 2011 (pour un montant de 490 milliards d'euros) et en février 2012 (pour un montant de 530 milliards d'euros). 


\section{Références}

V. Acharya, L. Pedersen, T. Philippon et M. Richardson [2010] : Regulating Systemic Risk, in Restoring Financial Stability: How to Repair a Failed System, V. Acharya et M. Richardson (éds) NYU Stern et Wiley, New York, pp. 283-304.

J.P. Allegret et C. Cornand [2006] : La politique de diffusion de l'information : la transparence des banques centrales est-elle toujours préférable ?, Revue française d'économie, 21 (1), pp. 87-126.

C. Antonin, C. Blot, P. Hubert, F. Labondance, C. Mathieu, C. Rifflart et V. Touzé [2013] : Politiques monétaires : est-ce le début de la fin ?, Revue de l'OFCE / Analyse et prévisions, 130, pp. 225-262.

P. Bacchetta, C. Tille C. et E. van Wincoop [2012] : Self-Fullfilling Risk Panics, The American Economic Review, 102 (7), pp. 3674-3700.

P. Bacchetta, C. Tille C. et E. van Wincoop [2010]: Risk Panics: When Markets Crash for No Apparent Reason, Vox, 19 juillet.

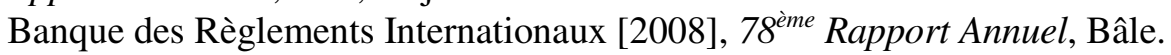

C. Bean [2009]: The Great Moderation, the Great Panic and the Great Contraction, Schumpeter Lecture-Annual congress of the European Economic Association, Barcelone, août.

B. Bernanke [2012]: Monetary Policy since the Onset of the Crisis, in The Changing Policy Landscape, Symposium Jackson Hole, The Federal Reserve Bank of Kansas City, pp. 1-22.

B. Bernanke [2009], The Crisis and the Policy Response, Stamp Lecture, London School of Economics, 13 janvier.

B. Bernanke, V. Reinhart et B. Sack [2004] : Monetary Policy Alternatives at the Zero Bound: An Empirical Assessment, Brookings Papers on Economic Activity, 2, pp. 1-100.

J. Bischof et H. Daske [2012] : Mandatory Supervisory Disclosure, Voluntary Disclosure, and Risk-Taking of Financial Institutions: Evidence from the EU-wide Stress-testing Exercises,

Conférence Disclosure, Transparency, and Financial Reporting in the Financial Services Industry, Federal Reserve Bank of New York, août.

A. Blinder [1998] : Central Banking in Theory and Practice, MIT Press, Cambridge Mass.

P. Bond et I. Goldstein [2012] : Government Intervention and Information Aggregation by Prices, Working paper, University of Pennsylvania.

C. Borio et M. Drehmann [2009] : Assessing the Risk of Banking Crises - Revisited, BIS quarterly review, mars, pp. 29-46.

M. Bouvard, P. Chaigneau et A. de Motta [2013] : Transparency in the Financial System: Rollover Risk and Crises, Conférence Bank liquidity, regulation, and transparency, Banque de France et Toulouse School of Economics, Paris, décembre.

N. Boyarchenko [2012]: Ambiguity Shifts and the 2007-2008 Financial Crisis, Journal of Monetary Economics, 59 (5), pp. 493-509.

E. Boz et E.G. Mendoza [2013] : Financial Innovation, the Discovery of Risk, and the U.S. Credit Crisis, Journal of Monetary Economics, à paraître.

R.J. Caballero et A. Krishnamurthy [2008] : Collective Risk Management in a Flight to Quality Episode, Journal of Finance, 63 (5), pp. 2195-2230.

A. Cardinali et J. Nordmark [2011] : How Informative are Bank Stress Tests? Bank Opacity in the European Union, Lund University.

M. Cecioni, G. Ferrero et A. Secchi [2011], Unconventional Monetary Policy in Theory and in Practice, Bank of Italy, Occasional papers, 102, septembre.

R. Clarida [2012] : What Has - and Has Not - Been Learned about Monetary Policy in a Low Inflation Environment? A Review of the 2000s, Journal of Money, Credit and Banking, 44 (1), pp. 123-140.

B. Couré [2013] : The Usefulness of Forward Guidance, Speech before the Money Marketeers Club of New York, New York, 26 septembre.

C. Cornand et C. Gimet [2012] : The 2007-2008 Financial Crisis: Is there Evidence of Disaster Myopia?, Emerging Markets Review, 13 (3), pp. 301-315.

S. Das [2011] : The EBA Stress Tests - Not the Real Thing, Eurointelligence.

G. Eggertsson G. et M. Woodford [2003] : The Zero Bound On Interest Rates and Optimal Monetary Policy, Brookings Papers on Economic Activity, 1, pp. 139-235. 
A. Ellahie [2012] : Bank Stress Tests and Information Asymmetry, Annual Meeting and Conference on Teaching and Learning Accounting, American Accounting Association, Washington D.C., août.

W.B. English, J.D. López-Salido, et R.J. Tetlow [2013] : The Federal Reserve's Framework for Monetary Policy, Recent Changes and New Questions, $\mathbf{1 4}^{\text {th }}$ Jacques Polak annual research conference hosted by the International Monetary Fund Washington D.C., novembre.

European Banking Authority [2011a], EU-wide Stress Test: Methodological Note, Londres, mars.

European Banking Authority [2011b], EU-wide Stress Test: Methodological Note, Additional Guidance, Londres, juin.

Federal Reserve [2009a]: The Supervisory Capital Assessment Program: Design and Implementation, Federal Reserve, Washington DC, avril.

Federal Reserve [2009b] : The Supervisory Capital Assessment Program: Overview of Results, Federal Reserve, Washington DC, mai.

Federal Reserve [2012] : Comprehensive Capital Analysis and Review 2012: Methodology and Results for Stress Scenario Projections, Board of Governors of the Federal Reserve System, Washington DC, mars.

M. Falagiarda et S. Reitz [2013] : Announcements of ECB Unconventional Programs: Implications for the Sovereign Risk of Italy, Kiel Institute for the World Economy, Working papers, 1866, août.

M. Flannery, S. Kwan et M. Nimalendran [2013] : The 2007-09 Financial Crisis and Bank Opaqueness, Journal of Financial Intermediation, 22 (1), pp. 55-84.

Fonds Monétaire International [2008], Global financial stability report, containing systemic risks and restoring financial soundness, avril, Washington DC.

A. Fracasso, H. Genberg et C. Wyplosz [2003] : How do Central Banks Talk? An Evaluation of Inflation Targeting Centrl Banks, Geneva report on the world economy, Special report 2, ICMBCEPR.

M. Fratzscher [2013] : Banques centrales : les inconvénients de la communication prospective "forward guidance", Le Cercle - Les Echos.

P. Gai et H.S. Shin [2003] : Transparency and Financial Stability, Financial Stability Review, 15, pp. 91-98.

J. Gagnon, M. Raskin, J. Remache et B. Sack [2011] : Large-Scale Asset Purchases by the Federal Reserve : Did They Work?, Federal Reserve Bank of New York, Economic Policy Review, mai, pp. 41-59.

GAO [2010] : Troubled Asset Relief Program: Bank Stress Test Offers Lessons as Regulators Take Further Actions to Strengthen Supervisory Oversight, US Government Accountability Office, Washington DC, septembre.

W. Gick et T. Pausch [2012] : Optimal Disclosure of Supervisory Information in the Banking Sector, Mimeo.

I. Goldstein et H. Sapra [2012] : Should Banks' Stress Test Results be Disclosed? An Analysis of the Costs and Benefits, The Wharton School - University of Pennsylvania.

I. Goldstein et Y. Leitner [2013] : Stress Tests and Information Disclosure, Federal Reserve Bank of Philadelphia, Working paper, 13-26, juin.

G.B. Gorton [2008] : The Panic of 2007, in Maintaining stability in a changing financial system, Jackson Hole Conference, Federal Reserve Bank of Kansas City, pp. 131-262.

G.B. Gorton [2009a] : The subprime panic, European Financial Management, 15 (1), pp. 10-46.

G.B. Gorton [2009b] : Information, Liquidity, and the [Ongoing] Panic of 2007, The American Economic Review, 99 (2), pp. 567-72.

G.B. Gorton et G. Ordonez [2012] : Collateral Crises, NBER working paper, 17771, janvier.

J.M. Guttentag et R.J. Herring [1986] : Disaster Myopia in International Banking, Essays in International Finance, 164, Princeton University Press.

S.G. Hanson et A. Sundera [2013] : Are there Too Many Safe Securities? Securitization and the Incentives for Information Production, Journal of Financial Economics, 108 (3), pp. 565-584

A.G. Haldane [2009]: Why Banks Failed the Stress Test, Speech given at the Marcus-Evans Conference on Stress-Testing, février. 
R.J. Herring [1998] : Banking Disasters: Causes and Preventative Measures, Lessons Derived from the U.S. Experience, in Preventing Bank Crises - Lessons from Recent Global Bank Failures, G. Jr. Caprio, W.C. Hunter, G.G. Kaufman et D.M. Leipziger (éds), pp. 209-236.

D. Kahneman, P. Slovic et A. Tversky [1982] : Judgment under Uncertainty: Heuristics and Biases. New York: Cambridge University Press.

J.M. Keynes [1936] : The General Theory of Employment, Interest, and Money, McMillan, Cambridge University Press.

C. Kool et D. Thornton [2012] : How Effective is Central Forward Guidance? Federal Reserve Bank of Saint Louis Working paper series, 2012-063A, décembre.

A. Krishnamurthy et A. Vissing-Jorgensen [2011] : The Effects of Quantitative Easing on Interest Rates: Channels and Implications for Policy, Brookings Papers Economic Activity, 2, pp. 215265.

P.R. Krugman [1998] : It's Baaack: Japan's Slump and the Return of the Liquidity Trap, Brookings Papers on Economic Activity, 2, pp. 137-206.

Y. Leitner [2012] : Inducing Agents to Report Hidden Trades: A Theory of an Intermediary, Review of Finance, 16 (4), pp. 1013-1042.

H.P Minsky [1977] : A Theory of Systemic Fragility, in Financial Crises, E.I. Altman et A.W. Sametz (éds.), Wiley, New York, pp. 138-152.

S. Morris S. et H.S. Shin [2002], Social Value of Public Information, The American Economic Review, 92 (5), pp. 1522-1534.

M. Pagano et P. Volpin [2012] : Securitization, Transparency, and Liquidity, The review of financial studies, 25 (8), pp. 2416-2453.

C. Pattipeilohy, J.W. van den End, M. Tabbae, J. Frost et J. de Haan [2013] : Unconventional Monetary Policy of the ECB during the Financial Crisis: An Assessment and New Evidence, De Nederlandsche Bank, DNB Working Paper, 381, Mai.

S. Peristiani, D.P. Morgan et V. Savino [2010] : The Information Value of the Stress Test and Bank Opacity, Federal Reserve Bank of New York Staff Reports, 460, juillet.

G. Petrella et A. Resti [2013] : Supervisors as Information Producers: Do Stress Tests Reduce Bank Opaqueness?, Journal of Banking and Finance, 37 (12), pp. 5406-5420.

C.M. Reinhart, et K.S. Rogoff [2009], This Time is Different: Eight Centuries of Financial Folly, Princeton University Press.

C. Romer et D. Romer [2000], Federal Reserve Private Information and the Behavior of Interest Rates, The American Economic Review, 90 (3), pp. 429-457.

T. Schuermann [2012] : Stress Testing Banks, Report Prepared for the Committee on Capital Markets Regulation, New York.

J. Shapiro et D. Skeie [2012] : Information Management in Banking Crises, CEPR Discussion Paper, DP9612, août.

F. Spargoli [2012] : Banks' Recapitalization and the Information Value of a Stress Test in a Crisis, Mimeo.

L. Svensson [2010], Policy Expectations and Policy Evaluations: The Role of Transparency and Communication, Sveriges Riksbank Economic Review, 1/2010, pp. 43-78.

C. Walsh [2008] : Announcements and the Role of Policy Guidance, Federal Reserve Bank of St. Louis Review, 90 (4), pp. 421-442.

N. Williams [2012] : Financial Instability via Adaptive Learning, University of Wisconsin, Mimeo. M. Woodford [2005] : Central Bank Communication and Policy Effectiveness, in The Greenspan era : lessons for the future, Symposium Jackson Hole, The Federal Reserve Bank of Kansas City, pp. 399-474.

M. Woodford [2013] : Methods of Policy Accommodation at the Interest-Rate Lower Bound, in Global Dimensions of Unconventional Monetary Policy, Symposium Jackson Hole, The Federal Reserve Bank of Kansas City, pp. 185-288.

J.L. Yellen [2011], Unconventional Monetary Policy and Central Bank Communications, The U.S. Monetary Policy Forum New York, New York, février.

J.L. Yellen [2013], Communication in Monetary Policy, Remarks at Society of American Business Editors and Writers, 50 ${ }^{\text {th }}$ Anniversary Conference, Washington, D.C., 4 avril. 